\title{
$k$-essence scalar dark matter solitons around supermassive black holes
}

\author{
Philippe Brax and Patrick Valageas (1) \\ Université Paris-Saclay, CNRS, CEA, Institut de physique théorique, 91191 Gif-sur-Yvette, France
}

Jose A. R. Cembranos $\odot$

Departamento de Física Teórica and IPARCOS, Universidad Complutense de Madrid, E-28040 Madrid, Spain

(Received 24 January 2020; accepted 19 February 2020; published 5 March 2020)

\begin{abstract}
We consider scalar dark-matter models where the theory has a shift symmetry only broken by the scalar mass term. We restrict ourselves to $k$-essence kinetic terms where the shift symmetric part of the Lagrangian is a function of the first derivatives of the scalar field only. When the scalar mass is much larger than the inverse of the astrophysical time and length scales of interest, these models provide a description of dark-matter equivalent to the one given by theories with only polynomial interactions, in the low-amplitude regime where the self-interactions are small contributions to the Lagrangian. In this regime and in the nonrelativistic limit, which apply on large galactic scales, scalar clouds form solitons with a finite core. This provides an adequate model for dark-matter halos with no singular behavior. Close to the center of galaxies, where a supermassive black hole $(\mathrm{BH})$ resides, we analyze the scalar-field distribution and the fate of the dark-matter soliton when subject to the BH gravitational attraction. We show that the scalar-field profile around such a central BH can be described by new oscillatory solutions of a modified Klein-Gordon equation, which generalize the harmonic oscillations of free scalar dark matter in a flat environment and the Jacobi elliptic functions of the $\phi^{4}$ model. Moreover, we find that, depending on the form of the $k$-essence kinetic term, regular solutions can be constructed or not, which connect the relativistic ingoing wavelike profile of the scalar field at the $\mathrm{BH}$ horizon to the nearly static nonrelativistic soliton at large distance. These profiles have a constant flux and represent the slow infall of scalar matter into the $\mathrm{BH}$. We show that this regular behavior is only possible for $k$-essence functions that satisfy the usual conditions for the absence of ghosts and gradient instabilities, together with a new restriction on the growth of the kinetic function $K(X)$ for large argument. It turns out that the same conditions of stability guarantee that quantum corrections are tamed, provided that the mass of the scalar field is less than $10^{-3} \mathrm{eV}$ and the strong coupling scale of the model $\Lambda$ is much larger than the scalar mass.
\end{abstract}

DOI: 10.1103/PhysRevD.101.063510

\section{INTRODUCTION}

Conventional descriptions of dark matter involving heavy particles with weak interactions (WIMPs) have failed to show up so far in all experiments tracking them from astrophysical scales, i.e., indirect detection, to the laboratory, i.e., direct detection and production at accelerators. Moreover, the confrontation between the predictions of such cold dark-matter (CDM) scenarios using large-scale computer simulations or analytical estimates is in tension with astrophysical observations [1-3]. The so-called "core-cusp" [4], "missing satellites" [5], or "too big to fail" [6] problems are well-known examples of these open questions that do not have a definitive answer. This has prompted the search for alternatives to the standard scenario. Axions [7-9] and axionlike particles [10] have been suggested and have particular features that are well documented. More generally, scalar fields, either fundamental or as an effective low energy description of underlying theories, have been extensively studied in the last decade. Particular emphasis has been put on fuzzy dark-matter models [11,12], where a very light scalar field could form a condensate whose average properties would coincide with the ones expected from dark matter for the formation of large scale structures [13-18], but present distinctive features for the behavior at small scales [1-6,16,19-25]. Indeed, one of the salient features of these models is the wavelike behavior of dark matter on galactic scales, following from the non-negligible role played by the so-called "quantum pressure." Such models require masses typically less than $10^{-21} \mathrm{eV}$ and could be in conflict with a host of astrophysical observations [26], such as the spectrum of the Lyman- $\alpha$ forest.

In this paper, we focus on scalar dark-matter models where the scalar field has a much larger mass [27]. In this regime, the quantum pressure can be neglected on galactic scales and the scalar self-interactions play a dominant role [17,24,28-32]. In particular, they can provide the repulsive 
pressure that balances the gravitational attraction, allowing for clouds of dark matter to be stable on large scales. Such clouds form solitonlike objects that are candidates for representing dark-matter halos with a finite core. This behavior is typically obtained for dark-matter scalar fields with a positive $\phi^{4}$ self-interaction. Moreover, as shown in [33], these solitons are long lived even when the supermassive black hole $(\mathrm{BH})$ at the center of the halo is taken into account. Indeed, the lifetime of such objects is longer than the age of the Universe.

Here we consider models of scalar dark matter where the scalar mass term is complemented with $k$-essence kinetic terms [34]. On large scales and in the nonrelativistic limit, these models are equivalent to self-interacting models of scalars with polynomial interactions. We extend this analysis to the case where there is a supermassive $\mathrm{BH}$ at the center of the galaxies. In this case, the equivalence with polynomial models is more subtle; in particular, we show that regular dark-matter profiles with constant scalar fluxes, which must behave as ingoing waves close to the $\mathrm{BH}$ horizon, cannot always be connected to the solitonic solution at large radii. This happens for the $(\partial \phi)^{4}$ model, where the scalar field cannot sustain a large scalar cloud in the presence of the central $\mathrm{BH}$. We give conditions for the existence of regular solutions where the scalar profile exists and is regular from the $\mathrm{BH}$ horizon to spatial infinity. On top of the usual $k$-essence stability conditions for the absence of ghosts and gradient instabilities, we find that the growth of the $k$-essence function for large argument cannot be too steep. In this case, this also guarantees that the models are stable under quantum corrections, even though the model becomes nonlinear close to the $\mathrm{BH}$ horizon.

The paper is arranged as follows. In Sec. II, we describe the models of scalar dark matter with nonlinear kinetic terms and connect them in the nonrelativistic regime with theories that have nonlinear scalar potentials. In Sec. III, we present the nonlinear solutions to the modified Klein-Gordon equation and the constant flux solutions. In Sec. IV, we make the connection between the nonlinear solutions and the largeradius and nonrelativistic limits. We also consider the behavior close to the horizon. In Sec. V, we give the example of quartic Lagrangians for which constant flux solutions connected to stable solitons at large radii do not exist. We then discuss when global solutions exist in Sec. VI. Then, in Sec. VII, we give an explicit example of models for which constant flux solutions up to very large radii exist and the lifetime of the soliton is larger than the age of the Universe. In Sec. VIII, we discuss the quantum stability of these models. We finally conclude in Sec. IX.

\section{DARK-MATTER SCALAR FIELD WITH DERIVATIVE SELF-INTERACTIONS}

\section{A. Scalar-field action with nonstandard kinetic term}

In this paper, we investigate scenarios where the darkmatter scalar-field action is

$$
S_{\phi}=\int d^{4} x \sqrt{-g}\left[\Lambda^{4} K(X)-\frac{m^{2}}{2} \phi^{2}\right]
$$

where the normalized kinetic argument $X$ is given by

$$
X=-\frac{1}{2 \Lambda^{4}} g^{\mu \nu} \partial_{\mu} \phi \partial_{\nu} \phi,
$$

and we decompose the nonstandard kinetic term $K(X)$ as the sum of the standard term $X$ and a nonstandard nonlinear contribution $K_{\mathrm{I}}$,

$$
K(X)=X+K_{\mathrm{I}}(X) .
$$

We assume that $K_{\mathrm{I}}$ admits the small- $X$ expansion

$$
X \ll 1: K_{\mathrm{I}}(X)=\sum_{n \geq 2} \frac{k_{n}}{n} X^{n} .
$$

The scale $\Lambda$ plays the role of the strong coupling scale. We shall check that the models make sense quantum mechanically even when $X \gg 1$; see Sec. VIII.

As shown in [27], in the nonrelativistic and large-mass regime, where $K_{\mathrm{I}} \ll X$, the small nonlinear correction $K_{\mathrm{I}}$ is equivalent to a small nonlinear potential $V_{\mathrm{I}}$, with $V_{\mathrm{I}} \ll$ $m^{2} \phi^{2} / 2$ and

$$
V_{\mathrm{I}}(\phi)=\Lambda^{4} \sum_{n \geq 4} \frac{\lambda_{n}}{n} \frac{\phi^{n}}{\Lambda^{n}}
$$

with

$$
\lambda_{2 n}=-2 k_{n}\left(\frac{m^{2}}{2 \Lambda^{2}}\right)^{n}
$$

This result is obtained at leading order in the large-mass limit, when the dynamics are averaged over the fast oscillations $e^{i m t}$ driven by the zeroth-order quadratic Lagrangian $\Lambda^{4} X-m^{2} \phi^{2} / 2$.

In the case of a quartic derivative self-interaction, we obtain

$K_{\mathrm{I}}(X)=\frac{k_{2}}{2} X^{2}, \quad V_{\mathrm{I}}(\phi)=\frac{\lambda_{4}}{4} \phi^{4}, \quad \lambda_{4}=-k_{2} \frac{m^{4}}{2 \Lambda^{4}}$.

For positive $\lambda_{4}$, hence negative $k_{2}$, this gives rise to an effective pressure on small scales [27]. This leads to a nonzero Jeans length for the growth of cosmological structures, and in virialized halos the scalar field can relax to a static soliton, where the halo self-gravity is balanced by this effective pressure due to the (derivative) self-interaction. Therefore, in this paper we focus on the case

$$
\lambda_{4}>0, \quad k_{2}<0 .
$$




\section{B. Isotropic coordinates}

Throughout most of this paper, we work with isotropic coordinates and we consider static spherically symmetric configurations. Then, the metric can be written as

$$
d s^{2}=-f(r) d t^{2}+h(r)\left(d r^{2}+r^{2} d \vec{\Omega}^{2}\right) .
$$

We use natural units with $c=1$ throughout this paper.

The spacetime around the $\mathrm{BH}$ can be divided in three regions. First, from the Schwarzschild radius and up to a radius $r_{\mathrm{NL}}$, the metric is in the strong-gravity regime dominated by the supermassive $\mathrm{BH}$ gravity. Then, the metric functions $f(r)$ and $h(r)$ are given by the standard Schwarzschild metric, but written in the isotropic coordinates $(r, t)$ instead of the usual Schwarzschild coordinates $(\tilde{r}, t)$. This gives [35]

$$
\begin{gathered}
\frac{r_{s}}{4}<r<r_{\mathrm{NL}}: f(r)=\left(\frac{1-r_{s} /(4 r)}{1+r_{s} /(4 r)}\right)^{2}, \\
h(r)=\left(1+r_{s} /(4 r)\right)^{4} .
\end{gathered}
$$

Here, $r_{s}=2 \mathcal{G} M_{\mathrm{BH}}$ is the Schwarzschild radius of the $\mathrm{BH}$ of mass $M_{\mathrm{BH}}$,

$$
r_{s}=2 \mathcal{G} M_{\mathrm{BH}} \simeq\left(\frac{M_{\mathrm{BH}}}{10^{8} M_{\odot}}\right) 10^{-8} \mathrm{kpc}
$$

$r=r_{s} / 4$ is the Schwarzschild radius in the radial isotropic coordinate $r$, which is related to the usual Schwarzschild radial coordinate $\tilde{r}$ by [35]

$$
\tilde{r}>r_{s}, \quad r>\frac{r_{s}}{4}: \tilde{r}=r\left(1+\frac{r_{s}}{4 r}\right)^{2} .
$$

Second, beyond $r_{\mathrm{NL}}$ and up to $r_{\mathrm{sg}}$, the metric is in the weak-gravity regime while the gravitational potential remains dominated by the $\mathrm{BH}$ itself. This gives

$$
r>r_{\mathrm{NL}}: f(r)=1+2 \Phi, \quad h(r)=1-2 \Phi,
$$

with

$$
r_{\mathrm{NL}}<r \ll r_{\mathrm{sg}}: \Phi(r)=-\frac{r_{s}}{2 r}=-\frac{\mathcal{G} M_{\mathrm{BH}}}{r} .
$$

Third, beyond the radius $r_{\mathrm{sg}}$ the metric is also in the weak-gravity regime, as in Eq. (14), but the gravitational potential is dominated by the self-gravity of the dark-matter scalar-field cloud. Then, $\Phi$ is given by the scalar-field Poisson equation

$$
r \gg r_{\mathrm{sg}}: \nabla^{2} \Phi=4 \pi \mathcal{G} \rho_{\phi},
$$

where $\rho_{\phi}$ is the scalar-field energy density.

\section{Equations of motion}

In the static spherical metric (9), the scalar-field KleinGordon equation reads

$$
\frac{\partial}{\partial t}\left[K^{\prime} \frac{\partial \phi}{\partial t}\right]-\sqrt{\frac{f}{h^{3}}} \frac{1}{r^{2}} \frac{\partial}{\partial r}\left[\sqrt{f h} r^{2} K^{\prime} \frac{\partial \phi}{\partial r}\right]+f m^{2} \phi=0,
$$

where $K^{\prime}=d K / d X$ and

$$
X=\frac{1}{2 \Lambda^{4} f}\left(\frac{\partial \phi}{\partial t}\right)^{2}-\frac{1}{2 \Lambda^{4} h}\left(\frac{\partial \phi}{\partial r}\right)^{2} .
$$

\section{Large-radius soliton}

At large radii, $r \gg r_{\mathrm{sg}}$, the gravitational field is small and set by the self-gravity of the scalar cloud. Therefore, assuming the influence of the $\mathrm{BH}$ can indeed be neglected, we recover the solitonic solution of the dark-matter halo as analyzed in [27]. We briefly recall in this section their results, which we need to set the large-radius boundary conditions when we analyze the exact solution that takes into account the central $\mathrm{BH}$. In this nonrelativistic regime, we can write the real scalar field $\phi$ in terms of a complex scalar field $\psi$ as

$$
\phi=\frac{1}{\sqrt{2 m}}\left(e^{-i m t} \psi+e^{i m t} \psi^{\star}\right) .
$$

In the large-mass limit, where macroscopic momentum scales are much below $m$, this actually decomposes $\phi$ in a fast oscillation $e^{ \pm i m t}$, which is associated with the zeroth order of the Klein-Gordon equation (17), $\partial_{t}^{2} \phi+m^{2} \phi=0$, and a slow time and space dependent part $\psi(r, t)$, which is associated with the variation of gravitational potentials and matter densities on astrophysical time and length scales. Next, the dynamics of the complex field $\psi$ can be mapped to hydrodynamics problem through the Madelung transformation [36],

$$
\psi=\sqrt{\frac{\rho}{m}} e^{i s}, \quad \phi=\frac{\sqrt{2 \rho}}{m} \cos (m t-s),
$$

where $\rho$ plays the role of the scalar-field matter density while the velocity field $\vec{v}$ is defined from the phase $s$ by

$$
\vec{v}=\frac{\vec{\nabla} s}{m} .
$$

Then, the dynamics are governed by the continuity and Euler equations,

$$
\begin{gathered}
\dot{\rho}+\vec{\nabla} \cdot(\rho \vec{v})=0, \\
\dot{\vec{v}}+(\vec{v} \cdot \vec{\nabla}) \vec{v}=-\vec{\nabla}\left(\Phi+\Phi_{\mathrm{I}}\right),
\end{gathered}
$$


where $\Phi$ is the gravitational potential (16), where $\rho_{\phi}=\rho$, and $\Phi_{\mathrm{I}}$ is a repulsive self-interaction potential. In the quartic case it is given by [27]

$$
\Phi_{\mathrm{I}}(\rho)=\frac{\rho}{\rho_{a}} \quad \text { with } \quad \rho_{a} \equiv \frac{4 m^{4}}{3 \lambda_{4}}=\frac{8 \Lambda^{4}}{3\left|k_{2}\right|} .
$$

Here we neglected the "quantum pressure" $\Phi_{\mathrm{Q}}$, associated with the wavelike nature of the scalar field, because we consider large masses $m \gg 10^{-21} \mathrm{eV}$, beyond the ranges associated with fuzzy dark-matter scenarios. The pressure $\Phi_{\text {I }}$ associated with the self-interactions allows the scalar cloud to reach a hydrostatic equilibrium, where this repulsive self-interaction balances the self-gravity. This gives the soliton profile [27]

$\rho(r)=\rho_{\mathrm{sol}}(0) \frac{\sin \left(r / r_{a}\right)}{r / r_{a}}, \quad \Phi_{\mathrm{I}}(r)=\Phi_{\mathrm{I}, \mathrm{sol}}(0) \frac{\sin \left(r / r_{a}\right)}{r / r_{a}}$,

with $\vec{v}=0$ and

$$
r_{a}=\frac{1}{\sqrt{4 \pi \mathcal{G} \rho_{a}}}=\sqrt{\frac{3 \lambda_{4}}{2}} \frac{M_{\mathrm{Pl}}}{m^{2}}
$$

where we introduced the reduced Planck mass $M_{\mathrm{Pl}}^{2}=1 /(8 \pi \mathcal{G})$. The soliton has a flat inner core and a finite radius $R_{\mathrm{sol}}=\pi r_{a}$, which can reach galactic size depending on the value of $\lambda_{4}$. More precisely, we can also write (26) as

$$
\lambda_{4}=\left(\frac{r_{a}}{20 \mathrm{kpc}}\right)^{2}\left(\frac{m}{1 \mathrm{eV}}\right)^{4}
$$

The constraint that the scalar field behaves as pressureless dark-matter at the background level up to the radiation-matter equality, at redshift $z_{\mathrm{eq}}$, implies [27] $\lambda_{4} \lesssim(m / 1 \mathrm{eV})^{4}$; therefore, we actually have $r_{a} \lesssim 20 \mathrm{kpc}$.

Inside the soliton, the hydrostatic equilibrium condition in Eq. (23) gives $\vec{\nabla}\left(\Phi+\Phi_{\mathrm{I}}\right)=0$, and we have

$$
r \leq R_{\mathrm{sol}}: \Phi+\Phi_{\mathrm{I}}=\alpha,
$$

where $\alpha$ is a constant, given by the value of the Newtonian potential at the boundary of the soliton,

$$
\alpha=\Phi\left(R_{\mathrm{sol}}\right),
$$

as $\Phi_{\mathrm{I}}\left(R_{\text {sol }}\right)=0$. In terms of the scalar fields $\psi$ and $\phi$ this gives [27]

$$
\psi=\sqrt{\frac{\rho}{m}} e^{-i \alpha m t}, \quad \text { hence } s=-\alpha m t,
$$

and

$$
\phi=\frac{\sqrt{2 \rho}}{m} \cos [(1+\alpha) m t]
$$

\section{NONLINEAR GLOBAL SOLUTION}

\section{A. Oscillating solution in the large-mass limit}

As in [33], where we considered the case of a scalar field with a standard kinetic term and a self-interaction potential, we look for a solution in the large-mass limit. Then, the field oscillates with a very high frequency determined by $m$, if we only keep the zeroth-order terms that give the standard Klein-Gordon equation $\partial_{t}^{2} \phi+m^{2} \phi=0$. However, the nonlinearity associated with the higher-order kinetic factor $K_{\text {I }}$ transforms this harmonic oscillator into an anharmonic oscillator, with parameters that slowly change with radius as dictated by the radial derivative term. In a fashion similar to the case of the quartic potential studied in [33], we look for a solution of the nonlinear Klein-Gordon equation (17) of the form

$$
\phi(r, t)=\phi_{0}(r) \operatorname{ck}[\omega(r) t-\mathbf{Q}(r) \beta(r), \mu(r)] .
$$

Here $\operatorname{ck}(u, \mu)$ is the extension of the harmonic cosine $\cos (u)$, obtained for the free massive scalar field, and of the Jacobi elliptic function $\mathrm{cn}(u, k)$, obtained for the quartic potential [33], to the case of derivative self-interactions (the letter "k" refers to the "kinetic" nonlinearity). For $\mu=0$ we recover the harmonic cosine, $\operatorname{ck}(u, 0)=\cos (u)$, and for nonzero $\mu$ we have an anharmonic oscillator, associated with the kinetic factor $K_{\mathrm{I}}$ that adds nonlinear contributions to the Klein-Gordon equation. The factor $\mathbf{Q}(r)$ is defined as $\mathbf{Q}(r) \equiv \mathbf{Q}[\mu(r)]$, where $\mathbf{Q}(\mu)$ is the quarter of the period of the oscillator $\operatorname{ck}(u)$ for parameter $\mu$. It is introduced in (32) for future convenience, to simplify Eq. (38) below. Thus, $\mu$ and $\mathbf{Q}$ play the role of the modulus $k$ and the complete elliptic integral $\mathbf{K}$ that appears in the case of the quartic potential [33]. At this stage, $\operatorname{ck}(u, \mu)$ is not defined yet and it is determined below from the analysis of the nonlinear Klein-Gordon equation.

The expression (32) is understood as the leading-order approximation in the limit $m \rightarrow \infty$, where spatial gradients of the functions $\phi_{0}, \omega, \mathbf{Q}, \beta$, and $\mu$ are much below $m$ (i.e., $\partial_{r} \ll m$ ), whereas both $\omega$ and $\beta$ are of order $m$. Thus, the scalar field shows fast oscillations with time at each radius, at a frequency and a phase of order $m$, with a slow modulation in space of the oscillation characteristics. This behavior relies on the large separation of scales $\partial_{r} \ll m$, which in our case corresponds to $r_{s} \gg m$, as radial derivatives typically scale as $\partial_{r} \sim 1 / r \lesssim 1 / r_{s}$ beyond the horizon.

To ensure that spatial gradients do not increase with time, the scalar field must oscillate with the same frequency over all radii, with a common period $T=2 \pi / \omega_{0}$, where $\omega_{0}$ is 
the common angular frequency. Otherwise, there would be a secular growth with time of the phase difference between neighboring points, hence a secular growth of radial gradients. Since the period of the function $\operatorname{ck}(u)$ for parameter $\mu$ is $4 \mathbf{Q}$, this implies $\omega T=4 \mathbf{Q}$ and $\omega(r)$ is fully determined by the oscillatory parameter $\mu(r)$ as

$$
\omega(r)=\frac{2 \mathbf{Q}(r)}{\pi} \omega_{0} .
$$

As we check in Sec. IV B and Eq. (88) below, the common frequency $\omega_{0}$ must match the oscillation found at large radii in the soliton solution (31). This implies

$$
\omega_{0}=(1+\alpha) m
$$

As we see in Sec. III B, the oscillating function obeys the Fourier series (62) below. Substituting into Eq. (32) gives

$$
\phi=\phi_{0}(r) \sum_{n=0}^{\infty} a_{2 n+1}(r) \cos \left[(2 n+1)\left(\omega_{0} t-\pi \beta(r) / 2\right)\right],
$$

with $a_{2 n+1}(r) \equiv a_{2 n+1}[\mu(r)]$. Thanks to the relation (33), we can see that the scalar field shows a coherent nonlinear oscillation over all radii, at the common angular frequency $\omega_{0}$.

From Eq. (32), the time derivative of the scalar field is

$$
\frac{\partial \phi}{\partial t}=\phi_{0} \omega \frac{\partial \mathrm{ck}}{\partial u} .
$$

At leading order in the large- $m$ limit, the radial derivative reads from Eq. (35) as

$\frac{\partial \phi}{\partial r} \simeq \phi_{0} \sum_{n=0}^{\infty} a_{2 n+1}(2 n+1) \frac{\pi \beta^{\prime}}{2} \sin \left[(2 n+1)\left(\omega_{0} t-\frac{\pi \beta}{2}\right)\right]$,

where $\beta^{\prime}=d \beta / d r$. Here we only kept the term of order $m$, as we assume that $\phi_{0}, \mu$, and $\beta$ are slow functions of $r$, but $\beta$ is of order $m$. Thus, the factor $\beta^{\prime}$ yields an additional power of $m$ as compared with $\phi_{0}^{\prime}$ or $a_{2 n+1}^{\prime}$. Comparing with the Fourier series of $\frac{\partial \mathrm{ck}}{\partial u}$, obtained from Eq. (62) below, this gives

$$
\frac{\partial \phi}{\partial r} \simeq-\phi_{0} \mathbf{Q} \beta^{\prime} \frac{\partial \mathrm{ck}}{\partial u}
$$

in this large- $m$ limit. The factor $\mathbf{Q}$ was introduced in Eq. (32) to simplify this radial derivative (the change $\beta \rightarrow$ $\beta / \mathbf{Q}$ would change the factor $\mathbf{Q} \beta^{\prime}$ above to $\left.\beta^{\prime}-\beta \mathbf{Q}^{\prime} / \mathbf{Q}\right)$. Indeed, if we had written $\phi=\phi_{0} \operatorname{ck}[\omega t-\beta, \mu]$, we would have found that a slow radial change of $\mu$, hence of the period 4Q, generates a leading-order change of the phase of the oscillation and must be taken into account. This effect is automatically taken care of by renormalizing the phase $\beta$ by the quarter of period $\mathbf{Q}$ in Eq. (32).

In this approximation, the kinetic term $X$ of Eq. (18) reads

$$
X=\frac{\phi_{0}^{2}}{2 \Lambda^{4} f}\left[\omega^{2}-\frac{f}{h}\left(\mathbf{Q} \beta^{\prime}\right)^{2}\right]\left(\frac{\partial \mathrm{ck}}{\partial u}\right)^{2}
$$

and the nonlinear Klein-Gordon equation (17) becomes

$$
\left[\omega^{2}-\frac{f}{h}\left(\mathbf{Q} \beta^{\prime}\right)^{2}\right]\left(1+\tilde{K}_{\mathrm{I}}\right) \frac{\partial^{2} \mathrm{ck}}{\partial u^{2}}+f m^{2} \mathrm{ck}=0,
$$

where we defined

$$
\tilde{K}_{\mathrm{I}}(X) \equiv K_{\mathrm{I}}^{\prime}+2 X K_{\mathrm{I}}^{\prime \prime}
$$

where the prime denotes the derivative with respect to $X$. If the self-interaction term $\tilde{K}_{\mathrm{I}}$ vanishes we recover the harmonic oscillator. For nonzero self-interaction, we obtain an anharmonic oscillator, with a derivative nonlinearity. The kinetic argument $X$ of Eq. (39) can be decomposed in a time-independent prefactor, with a slow radial dependence, and a fast oscillatory term. Thus, we define the prefactor $\mu(r)$ by

$$
\mu(r) \equiv \frac{\phi_{0}^{2}}{2 \Lambda^{4} f}\left[\omega^{2}-\frac{f}{h}\left(\mathbf{Q} \beta^{\prime}\right)^{2}\right]
$$

so that we have

$$
X=\mu(r)\left(\frac{\partial \mathrm{ck}}{\partial u}\right)^{2}
$$

Now, let us define an oscillatory function $\operatorname{ck}(u, \mu)$, of argument $u$ and parameter $\mu$, by the differential equation

$$
\frac{\partial^{2} \mathrm{ck}}{\partial u^{2}}+\mathrm{ck}+\tilde{K}_{\mathrm{I}}\left[\mu\left(\frac{\partial \mathrm{ck}}{\partial u}\right)^{2}\right] \frac{\partial^{2} \mathrm{ck}}{\partial u^{2}} \equiv 0,
$$

and the initial conditions

$$
\operatorname{ck}(0, \mu) \equiv 1, \quad \frac{\partial \mathrm{ck}}{\partial u}(0, \mu) \equiv 0 .
$$

From Eq. (4) we have $\tilde{K}_{\mathrm{I}} \rightarrow 0$ for $X \rightarrow 0$. Therefore, in the limit $\mu \rightarrow 0$ the nonlinear differential equation (44) simplifies to the linear harmonic oscillator, $\frac{\partial^{2} \mathrm{ck}}{\partial u^{2}}+\mathrm{ck}=0$, and with the initial conditions (45) we recover the cosine function,

$$
\operatorname{ck}(u, 0)=\cos (u)
$$

The initial conditions (45) do not entail any loss of generality. They mean that $\mathrm{ck}(u)$ oscillates over the range 


$$
-1 \leq \mathrm{ck} \leq 1
$$

and it starts at a maximum at $u=0$. The normalization to unity of the amplitude of ck simply sets the normalization of the amplitude $\phi_{0}$ in Eq. (32), while the choice $u=0$ for a maximum sets an integration constant for the phase $\beta$ or the origin of time $t$. Also, the choice of unity for the first two coefficients in the differential equation (44) does not lead to a loss of generality. It sets the normalization of the argument $u$, that is, the period $4 \mathbf{Q}$ of the oscillator.

Then, comparing the definition (44) with the nonlinear Klein-Gordon equation (40), we can see that this equation of motion is satisfied if $\operatorname{ck}(u, \mu)$ is the function defined in Eq. (44), provided we have

$$
f m^{2}=\omega^{2}-\frac{f}{h}\left(\mathbf{Q} \beta^{\prime}\right)^{2},
$$

and the parameter $\mu$ of the oscillator (44) is set to the value $\mu(r)$ of Eq. (42). Combining with Eqs. (33) and (42), we obtain

$$
\begin{aligned}
\frac{\pi^{2} f}{4 h} \beta^{\prime 2} & =\omega_{0}^{2}-\frac{\pi^{2} m^{2} f}{4 \mathbf{Q}^{2}}, \\
\phi_{0}^{2} & =\frac{2 \Lambda^{4}}{m^{2}} \mu .
\end{aligned}
$$

These two equations take the same form as Eqs. (62) and (63) obtained in [33] for the case of a quartic potential. For a given radial function $\mu(r)$, they provide the phase $\beta(r)$ and the amplitude $\phi_{0}(r)$. This fully determines the oscillating solution (32), as the frequency $\omega(r)$ is given by Eq. (33) and $\mathbf{Q}(r)$ is determined by $\mu(r)$ as the quarter of period of the oscillator (44). Equation (50) provides at once the constraint

$$
\mu \geq 0
$$

We can see in Eq. (50) that for low scalar-field amplitudes, $\phi_{0} \rightarrow 0$, we recover the harmonic oscillator as $\mu \rightarrow 0$. This corresponds to the nonrelativistic and smallfield limit, found for instance for the soliton solution (31), where the higher-order contributions to the scalar-field Lagrangian are small, $K_{\mathrm{I}} \ll X$, and the Klein-Gordon equation reduces to the harmonic oscillator at leading order.

Equation (49) appears as the generalization of the Euler equation at leading order, $\pi \beta^{\prime} /(2 m)$ playing the role of the radial velocity $v_{r}=m^{-1} d s / d r$ and $\pi \beta / 2$ the role of the phase $s$. Using Eqs. (34) and (49) we can write

$$
v_{r} \equiv \frac{\pi \beta^{\prime}}{2 m}=-\sqrt{\frac{h}{f}} \sqrt{(1+\alpha)^{2}-\frac{\pi^{2} f}{4 \mathbf{Q}^{2}}} .
$$

We refine this analogy below.

\section{B. Nonlinear oscillator}

We investigate the behavior of the anharmonic oscillator (44). This equation of motion can be integrated once, after multiplying by $2 \frac{\partial \mathrm{ck}}{\partial u}$. With the initial conditions (45), this gives

$$
\left(\frac{\partial \mathrm{ck}}{\partial u}\right)^{2}+\mathrm{ck}^{2}+\frac{1}{\mu} G_{\mathrm{I}}\left[\mu\left(\frac{\partial \mathrm{ck}}{\partial u}\right)^{2}\right]=1,
$$

where we introduced the function $G_{\mathrm{I}}(X)$ defined by

$$
G_{\mathrm{I}}(0) \equiv 0, \quad G_{\mathrm{I}}^{\prime} \equiv \tilde{K}_{\mathrm{I}}=K_{\mathrm{I}}^{\prime}+2 X K_{\mathrm{I}}^{\prime \prime}
$$

hence

$$
G_{\mathrm{I}}(X)=2 X K_{\mathrm{I}}^{\prime}(X)-K_{\mathrm{I}}(X) .
$$

This corresponds to the conservation of energy of the nonlinear oscillator, which oscillates over the range $-1 \leq$ $\operatorname{ck}(u) \leq 1$ with a period that we denote by $4 \mathbf{Q}(\mu)$. For small $\mu$ the term $G_{\mathrm{I}}$ is a small correction and $\operatorname{ck}(u)$ closely follows $\cos (u)$. For larger $\mu$, the higher-order contribution $G_{\text {I }}$ becomes important and the oscillations are more strongly deformed. Depending on the function $G_{I}$ the periodic oscillatory behavior may eventually disappear. Introducing the function $G(X)$ by

$$
G(X) \equiv X+G_{\mathrm{I}}(X)=2 X K^{\prime}(X)-K(X),
$$

the conservation equation (53) can be inverted as

$$
\left(\frac{\partial \mathrm{ck}}{\partial u}\right)^{2}=\frac{1}{\mu} G^{-1}\left[\mu\left(1-\mathrm{ck}^{2}\right)\right],
$$

where $G^{-1}$ is the inverse function of $G, G\left[G^{-1}(y)\right]=y$. For small $X$ we have $G(X) \simeq X$ and $G^{-1}(y) \simeq y$. Thus, near the maximum $\operatorname{ck}(u=0)=1$ we have $\left(\frac{\partial \mathrm{ck}}{\partial u}\right)^{2} \simeq 1-\mathrm{ck}^{2}$. To the right of the maximum, $u \gtrsim 0$, the function $\mathrm{ck}$ decreases below unity with a negative slope given by $\frac{\partial \mathrm{ck}}{\partial u} \simeq-\sqrt{1-\mathrm{ck}^{2}}$. We can build the periodic function $\operatorname{ck}(u)$ from its first quarter of period, where $0 \leq u \leq \mathbf{Q}$ and $1 \geq \mathrm{ck} \geq 0$, if we can solve the equation (57) until the point $\mathrm{ck}=0$ in a finite time $u=\mathbf{Q}$. For a given parameter $\mu \geq 0$, this requires that $G^{-1}(y)$ is well defined and positive over $0 \leq y \leq \mu$. Since $G^{-1}$ is defined from $G(X)$ with $X \geq 0$, starting from $G \simeq X$ at low $X$, we can see that $G^{-1}$ is positive from the parametric representation $\left\{y, G^{-1}\right\}=$ $\{G, X\}$. Moreover, it is well defined up to $\mu$ if $G(X)$ is monotonically increasing over $0 \leq X \leq X_{\mu}$, where $X_{\mu}$ is defined by $G\left(X_{\mu}\right) \equiv \mu$. From Eq. (56) this implies

$$
0 \leq X \leq X_{\mu}: G^{\prime}(X)=K^{\prime}+2 X K^{\prime \prime}>0 .
$$

Then, the dynamics can be solved by quadrature, 


$$
0 \leq \mathrm{ck} \leq 1: u=\int_{\mathrm{ck}}^{1} d \psi \sqrt{\frac{\mu}{G^{-1}\left[\mu\left(1-\psi^{2}\right)\right]}}
$$

For $\mu \rightarrow 0$ we recover the arc cosine function. Then, the quarter $\mathbf{Q}$ of the period is given by

$$
\mathbf{Q}(\mu)=\int_{0}^{1} d \psi \sqrt{\frac{\mu}{G^{-1}\left[\mu\left(1-\psi^{2}\right)\right]}} .
$$

Therefore, we can build an oscillatory solution (32) for the scalar field $\phi(r, t)$ if for all values $\mu(r)$ that are reached beyond the horizon the function $\mathbf{Q}(\mu)$ defined by Eq. (60) is well defined and finite. As for the cosine, we can build $\operatorname{ck}(u)$ over all real $u$ from the first quarter of period, $0 \leq u \leq \mathbf{Q}$. We first extend up to the first minimum, $\mathbf{Q} \leq u \leq 2 \mathbf{Q}$, with $\operatorname{ck}(2 \mathbf{Q}-u)=-\operatorname{ck}(u)$. Second, we extend to the first minimum on the left, at $u=-2 \mathbf{Q}$, with $\operatorname{ck}(-u)=\operatorname{ck}(u)$. Third, we extend from $-2 \mathbf{Q} \leq u \leq 2 \mathbf{Q}$ to all real $u$ with the periodicity $\operatorname{ck}(u+4 \mathbf{Q})=\operatorname{ck}(u)$. In other words, like the cosine, the periodic function $\operatorname{ck}(u)$ is even, of period $4 \mathbf{Q}$, and verifies $\operatorname{ck}(2 \mathbf{Q}-u)=-\operatorname{ck}(u)$,

$$
\begin{aligned}
\operatorname{ck}(-u) & =\operatorname{ck}(u), \quad \operatorname{ck}(u+4 \mathbf{Q})=\operatorname{ck}(u), \\
\operatorname{ck}(2 \mathbf{Q}-u) & =-\operatorname{ck}(u) .
\end{aligned}
$$

This implies that its Fourier series takes the form

$$
\operatorname{ck}(u, \mu)=\sum_{n=0}^{\infty} a_{2 n+1}(\mu) \cos \left[(2 n+1) u \frac{2 \pi}{4 \mathbf{Q}}\right]
$$

From Eq. (4) we obtain the series expansions

$$
\begin{aligned}
& X \rightarrow 0: G(X)=X+\frac{3}{2} k_{2} X^{2}+\ldots, \\
& y \rightarrow 0: G^{-1}(y)=y-\frac{3}{2} k_{2} y^{2}+\ldots,
\end{aligned}
$$

and substituting into Eq. (60) yields

$$
\mathbf{Q}(\mu)=\frac{\pi}{2}\left(1+\frac{3 k_{2}}{8} \mu+\ldots\right) .
$$

\section{Steady state and constant flux}

As we have seen above, Eqs. (49) and (50) determine the scalar-field solution (32) as a function of $\mu(r)$, but we have not specified the radial profile of $\mu(r)$ yet. This is provided by the condition of constant radial flux, after averaging over the fast oscillation of angular frequency $\omega_{0}$. The relativistic counterpart of the continuity equation is the component $\nu=0$ of the conservation equations $\nabla_{\mu} T_{\nu}^{\mu}=0$. The energy-momentum tensor of the scalar field $\phi$ with the action (1) reads

$$
\rho_{\phi} \equiv-T_{0}^{0}=\frac{K^{\prime}}{f}\left(\frac{\partial \phi}{\partial t}\right)^{2}-\Lambda^{4} K+\frac{m^{2}}{2} \phi^{2},
$$

and

$$
T_{0}^{r}=\frac{K^{\prime}}{h} \frac{\partial \phi}{\partial r} \frac{\partial \phi}{\partial t}
$$

The conservation equation $\nabla_{\mu} T_{0}^{\mu}=0$ becomes

$$
\frac{\partial \rho_{\phi}}{\partial t}-\frac{1}{\sqrt{f h^{3}} r^{2}} \frac{\partial}{\partial r}\left[\sqrt{f h^{3}} r^{2} T_{0}^{r}\right]=0
$$

At leading order, this continuity equation is satisfied by the balance between fast oscillatory terms. However, to ensure that secular terms do not appear at subleading order, we clearly require that in the steady state the averaged value of $\rho_{\phi}$ over one oscillation period should not depend on time. This gives the condition of constant flux $F$,

$F=-\sqrt{f h^{3}} r^{2}\left\langle T_{0}^{r}\right\rangle=\sqrt{f h} r^{2} \phi_{0}^{2} \omega \mathbf{Q} \beta^{\prime}\left\langle K^{\prime}\left(\frac{\partial \mathrm{ck}}{\partial u}\right)^{2}\right\rangle$,

where $\langle\ldots\rangle$ denotes the average over one oscillation period $T=2 \pi / \omega_{0}$ and we used Eqs. (36) and (38).

Using Eqs. (33), (34), (50), and (52), we can write the flux in terms of $\mu(r)$,

$$
F=F_{s} x^{2} h\left(\frac{2 \mathbf{Q}}{\pi}\right)^{2} C_{\mu} \mu \sqrt{1-\frac{\pi^{2} f}{(1+\alpha)^{2} 4 \mathbf{Q}^{2}}}
$$

where we defined the dimensionless radial coordinate

$$
x=\frac{r}{r_{s}} \geq \frac{1}{4}
$$

the characteristic flux

$$
F_{s}=-r_{s}^{2} 2 \Lambda^{4}(1+\alpha)^{2},
$$

and the average

$$
C_{\mu}=\left\langle K^{\prime}\left(\frac{\partial \mathrm{ck}}{\partial u}\right)^{2}\right\rangle
$$

Using Eq. (57) we obtain

$$
\begin{aligned}
C_{\mu}= & \frac{1}{\mathbf{Q}} \int_{0}^{\mathbf{Q}} d u K^{\prime}\left(\frac{\partial \mathrm{ck}}{\partial u}\right)^{2} \\
= & \frac{1}{\mathbf{Q} \sqrt{\mu}} \int_{0}^{1} d \psi \sqrt{G^{-1}\left[\mu\left(1-\psi^{2}\right)\right]} \\
& \times K^{\prime}\left[G^{-1}\left[\mu\left(1-\psi^{2}\right)\right]\right] .
\end{aligned}
$$


Then, for a given value of the flux $F$, the profile $\mu(r)$ of this steady state solution is determined by Eq. (70). The parameter $\mu$ at each radius, $x=r / r_{s}$, is such that the right-hand side in Eq. (70) is equal to $F$.

From Eq. (65), we can see that for $k_{2}<0$ the period $4 \mathbf{Q}$ decreases for higher $\mu$ (at least until higher-order terms become relevant). On the other hand, for the square root in Eq. (70) to be well defined, its argument must be positive, which implies $\mathbf{Q}^{2}>\pi^{2} f /\left[4(1+\alpha)^{2}\right]$. Therefore, $\mathbf{Q}$ cannot be too small, which typically means that $\mu$ cannot be too large and must obey the upper bound $\mu_{+}$,

$$
0 \leq \mu \leq \mu_{+}(x) \quad \text { with } \quad \mathbf{Q}^{2}\left(\mu_{+}\right)=\frac{\pi^{2} f}{4(1+\alpha)^{2}} .
$$

Depending on the function $\mathbf{Q}(\mu)$ and the radius $r$, this upper bound may be finite or pushed to infinity. If it is finite, the flux $F(\mu, x)$ as a function of $\mu$ for fixed $x$ vanishes at both ends, $\mu=0$ and $\mu=\mu_{+}(x)$. Typically, as in the case of the quartic potential studied in [33], $F(\mu, x)$ shows a single peak between these two end points, so that at each radius there are two solutions $\mu_{1}<\mu_{2}$ to Eq. (70), associated with the crossing of the value $F$ along the left and right sides of the peak.

These two solutions are associated with low-velocity and high-velocity branches. The boundary condition at large radii associated with the soliton described in Sec. II D selects the low-velocity branch (as the soliton corresponds to a static regime), while the boundary condition at the Schwarzschild radius selects the high-velocity branch (as near the horizon the self-interactions can no longer resist the $\mathrm{BH}$ gravity and the fluid falls inward with a relativistic velocity). Then, there is a unique critical value $F_{c}$ for the flux, such that these two branches connect at some intermediate radius $x_{\star}$ and the solution can smoothly switch from the low-velocity branch at large radii to the high-velocity branch at small radii. This gives rise to a picture that is similar to the unique transonic solution found in the case of the hydrodynamic infall of relativistic fluids into a $\mathrm{BH}$ [37].

\section{CONNECTING THE SMALL-FIELD LARGE- RADII REGIME TO THE BLACK HOLE HORIZON}

\section{A. Small- $\mu$ regime}

At large radii, the metric function $f(r)$ is close to unity, up to deviations of order $10^{-5}$ as in Eq. (14). The constant $\alpha$ is also of order $10^{-5}$ from Eq. (29), as this is the typical magnitude of the gravitational potential in galactic halos. Then, from the expansion (65) we can see that the bound (76) is reached for a small value of $\mu$, typically $\left|k_{2}\right| \mu_{+} \sim 10^{-5}$. Therefore, we investigate in this section the regime $\mu \ll 1$, where the function $\operatorname{ck}(u)$ is close to $\cos (u)$, as seen in (46), and $\mathbf{Q} \simeq \pi / 2, C_{\mu} \simeq 1 / 2$. Therefore, at large radii the global solution (32) becomes

$$
r \gg r_{s}: \phi \simeq \phi_{0}(r) \cos \left[\omega_{0} t-\pi \beta(r) / 2\right]
$$

and $\mu \ll 1$, assuming that $k_{2}$ is of order unity.

At lowest order over $\mu, \Phi$, and $\alpha$, the flux (70) reads

$$
\{\mu, \Phi, \alpha\} \ll 1: \frac{F}{F_{s}} \simeq \frac{x^{2}}{2} \mu \sqrt{2 \alpha-2 \Phi+3 k_{2} \mu / 4},
$$

where we used Eqs. (14) and (65). Then, the upper bound $\mu_{+}$introduced in (76), associated with the 0 of the square root, is given by (we recall that $k_{2}<0$ )

$$
r \gg r_{s}: \mu_{+}(r)=\frac{8(\alpha-\Phi)}{3\left|k_{2}\right|}=\frac{4(\alpha-\Phi) m^{4}}{3 \lambda_{4} \Lambda^{4}} .
$$

This explicitly shows that $\left|k_{2}\right| \mu \ll 1$ at large radii. From Eq. (43) we also have $X \lesssim \mu$ and $\langle X\rangle \simeq \mu / 2$. Then, the nonlinear contribution $K_{\mathrm{I}}$ to the kinetic term in the scalarfield Lagrangian is much smaller than the standard linear term $X$. This is the nonrelativistic regime, which can be described by the complex scalar field $\psi$ or the hydrodynamical picture $\{\rho, \vec{v}\}$ as in (20). This covers the soliton solution (25), at large radii $r \gg r_{\mathrm{sg}}$ dominated by the scalar cloud self-gravity, as well as the smaller radii $r_{\mathrm{NL}} \ll r<$ $r_{\mathrm{sg}}$ dominated by the $\mathrm{BH}$ gravity in the weak-field regime.

The flux (78) as a function of $\mu$, for a given radius $x$, vanishes at both ends of the allowed range $0 \leq \mu \leq \mu_{+}(x)$ and shows a single peak at the position $\mu_{\text {peak }}$ and height $F_{\text {peak }}$, with

$$
\mu_{\text {peak }}=\frac{2}{3} \mu_{+}, \quad \frac{F_{\text {peak }}}{F_{s}}=\frac{4 x^{2}}{3\left|k_{2}\right|}\left[\frac{2(\alpha-\Phi)}{3}\right]^{3 / 2} .
$$

In the weak-gravity regime (15) dominated by the $\mathrm{BH}$, we have $|\Phi| \gg|\alpha|$ and $\Phi \propto x^{-1}$. Therefore, in the range $r_{\mathrm{NL}} \ll$ $r \ll r_{\text {sg }}$ the peak height $F_{\text {peak }}$ grows with the radius as $F_{\text {peak }} \propto x^{1 / 2}$. At larger radii, $r_{\mathrm{sg}} \ll r \ll R_{\text {sol }}$, dominated by the scalar cloud gravity, $\Phi$ is almost constant and set by its value inside the soliton core, so that $F_{\text {peak }}$ grows with radius as $F_{\text {peak }} \propto x^{2}$. Thus, in agreement with Figs. 1 and 4 below, we find at large radii a universal behavior set by the weakfield and nonrelativistic regime and the first higher-order contribution $k_{2} X^{2} / 2$ to the Lagrangian kinetic term. The flux $F(\mu, x)$ shows a single peak with a height that grows with the distance from the central $\mathrm{BH}$.

As recalled above, this means that for a given value of the flux $F>0$, which defines the steady state solution, Eq. (78) has two solutions $\mu_{1}(x)$ and $\mu_{2}(x)$ at each radius $x$, with $0<\mu_{1}<\mu_{\text {peak }}<\mu_{2}<\mu_{+}$, provided that $F<F_{\text {peak }}$. They correspond to the crossing of the value $F$ along the two sides of the peak. Since the peak height $F_{\text {peak }}$ grows with the radius, these solutions are increasingly close to the end points 0 and $\mu_{+}$. In other words, as the factor $x^{2}$ becomes large in Eq. (78), either $\mu$ or $\sqrt{2 \alpha-2 \Phi+3 k_{2} \mu / 4}$ 


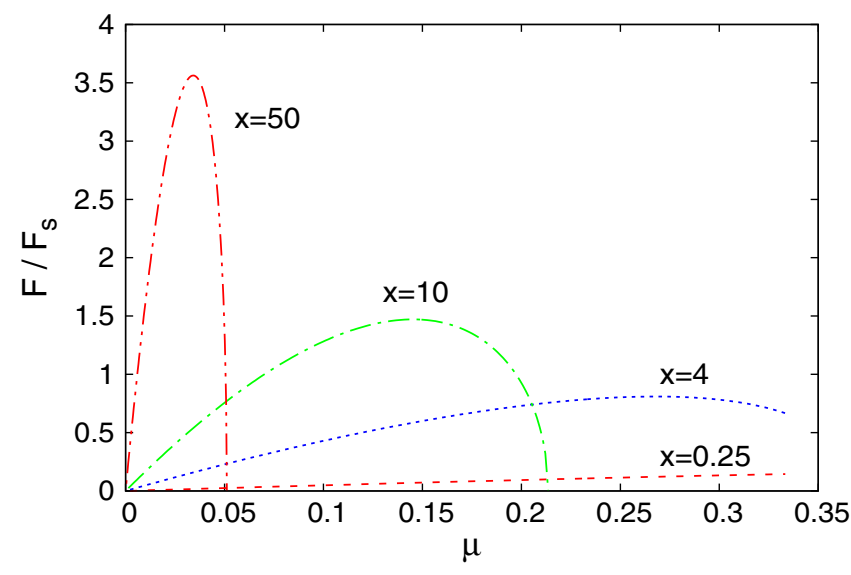

FIG. 1. Normalized flux $F(\mu, x) / F_{s}$ as a function of the oscillatory parameter $\mu$, for various values of the radial coordinate $x$, from Eq. (70) for the case of the quartic Lagrangian (96).

goes to 0 as $1 / x^{2}$. This gives the two asymptotic solutions $\mu_{1}<\mu_{2} \ll 1$,

$$
\begin{gathered}
x \gg 1: \mu_{1}(x)=\frac{\sqrt{2} F}{F_{s} \sqrt{\alpha-\Phi} x^{2}}+\ldots, \\
\mu_{2}(x) \simeq \frac{8(\alpha-\Phi)}{3\left|k_{2}\right|}-\frac{3\left|k_{2}\right|}{4}\left(\frac{F}{F_{s}(\alpha-\Phi) x^{2}}\right)^{2}+\ldots
\end{gathered}
$$

where the dots stand for higher-order terms over $1 / x^{2}$.

At lowest order in $\mu, \Phi$, and $\alpha$, the velocity $v_{r}$ of Eq. (52) reads

$$
x \gg 1: v_{r} \simeq-\sqrt{2 \alpha-2 \Phi+3 k_{2} \mu / 4} .
$$

As $\mu_{1} \ll \mu_{2}$, we can see that the left and right branches leads to the different behaviors

$$
\begin{aligned}
& x \gg 1: v_{r_{1}} \simeq-\sqrt{2(\alpha-\Phi)}, \\
& v_{r_{2}} \simeq-\frac{3\left|k_{2}\right| F}{4 F_{s}(\alpha-\Phi) x^{2}} \rightarrow 0 .
\end{aligned}
$$

Thus, $\mu_{1}$ is the high-velocity branch, as $v_{r_{1}}$ is of the order of the free-fall velocity, while $\mu_{2}$ is the low-velocity branch, as $v_{r_{2}}$ is much smaller and becomes negligible at large radii.

\section{B. Soliton boundary conditions}

We derive the large-radius boundary condition required by a matching to the soliton solution recalled in Sec. II D. At large radii but within the soliton radius, $r_{\text {sg }} \ll r \ll R_{\text {sol }}$, the scalar field is in the weak-gravity regime dominated by the scalar cloud mass and approaches the core of the soliton solution (25). As seen in the previous section, this also corresponds to the small- $\mu$ regime. The comparison of the expression (77) with Eq. (31) gives

$$
r_{\mathrm{sg}} \ll r \ll R_{\mathrm{sol}}: \phi_{0}(r)=\frac{\sqrt{2 \rho_{\mathrm{sol}}(0)}}{m}, \quad \beta \simeq 0,
$$

and Eq. (34), where $\alpha$ takes the value defined by the soliton solution (31). Indeed, as the soliton solution (31) corresponds to the hydrostatic equilibrium with $\vec{v}=0$, the velocity $\beta^{\prime}$ must become negligible at large radii in order to match with the soliton. The uniform angular frequency $\omega_{0}$ is also set by this large-radius boundary condition. As $|\alpha| \lesssim 10^{-5}$ from Eq. (29), $\omega_{0}$ remains very close to $m$.

We can now check that this is consistent with Eq. (49). Combining Eqs. (50) and (86) we obtain

$$
\mu=\frac{\rho}{\Lambda^{4}}=\frac{8}{3\left|k_{2}\right|} \Phi_{\mathrm{I}}
$$

where we introduced the repulsive potential defined in (24). Equation (49) with $\beta^{\prime}=0$ gives, at leading order in $\Phi$ and $\mu$,

$$
\omega_{0}=m\left(1+\Phi-3 k_{2} \mu / 8\right)=m\left(1+\Phi+\Phi_{\mathrm{I}}\right),
$$

where we used the series expansion (65) and in the last equality we used Eq. (87). Then, using Eq. (28) we recover Eq. (34). This shows that this large-radius asymptote is selfconsistent.

Using $\Phi_{\mathrm{I}}=\alpha-\Phi$ from Eq. (28), the comparison of Eq. (87) with Eq. (82) shows that the matching to the soliton solution selects the branch $\mu_{2}(x)$ at large radii,

$$
\mu=\mu_{2}(x) \text { for } r \gg r_{\mathrm{sg}} .
$$

This agrees with the fact that the branch $\mu_{2}(x)$ corresponds to the solution with negligible radial velocity, as shown in Eq. (85), which allows the matching to the static soliton.

\section{Boundary condition at the horizon}

Close to the horizon the self-interactions cannot counteract the BH gravity and the scalar field is in a free-fall regime where $v_{r} \sim-\sqrt{h / f}$ [33], with purely ingoing solutions and relativistic velocities. Therefore, we must reach the high-velocity branch $\mu_{1}(x)$,

$$
\mu=\mu_{1}(x) \quad \text { for } r \simeq r_{s} / 4 .
$$

This corresponds to the solution of Eq. (70), understood as an equation for $\mu$, that is on the left side of the peak of $F(\mu)$. From Eq. (52) we also recover $v_{r} \sim-\sqrt{h / f}$ near the horizon for this branch, as $f \rightarrow 0$ and the last square root becomes of order unity.

In the relativistic regime, $v_{r}$ can no longer be identified with a physical velocity (e.g., the velocity of particles of mass $m$ ) as the Euler equation (23) no longer applies. This is why it can go to infinity, whereas the velocity of an infalling particle measured by a distant observer would 
actually vanish at the horizon because of a strong redshift. We see in Sec. VII D that the divergence of $v_{r}$ at the horizon is only an artefact and that the scalar field $\phi$ remains well behaved down to the Schwarzschild radius.

\section{QUARTIC LAGRANGIAN}

We consider in this section the case of the quartic scalarfield Lagrangian (7), where only an $X^{2}$ term is added to the standard kinetic term. This provides a simple example where we cannot connect to the soliton at large radii and there is no steady solution with a slow infall into the $\mathrm{BH}$.

\section{A. Limited parameter range for the nonlinear oscillator}

In this quartic case, we have

$$
G(X)=X-\frac{3\left|k_{2}\right|}{2} X^{2},
$$

and

$$
0 \leq y \leq \frac{1}{6\left|k_{2}\right|}: G^{-1}(y)=\frac{1-\sqrt{1-6\left|k_{2}\right| y}}{3\left|k_{2}\right|} .
$$

Because $G(X)$ is only monotonically increasing on the finite interval $0 \leq X \leq 1 /\left(3\left|k_{2}\right|\right)$ and decreasing beyond, the inverse function $G^{-1}(y)$ is only defined over the finite range $0 \leq y \leq 1 /\left(6\left|k_{2}\right|\right)$. Following the analysis of Sec. III B, the differential equation (44) only admits a periodic oscillating solution defined for all real $u$ if $\mu<1 /\left(6\left|k_{2}\right|\right)$, when the quarter of period $\mathbf{Q}$ obtained in Eq. (60) is well defined. It now reads as

$$
\mathbf{Q}(\mu)=\int_{0}^{1} d \psi \sqrt{\frac{3\left|k_{2}\right| \mu}{1-\sqrt{1+6\left|k_{2}\right| \mu\left(\psi^{2}-1\right)}}} .
$$

For larger values of $\mu$, the function $\operatorname{ck}(u)$ displays a singularity at $\mathrm{ck}=\sqrt{1-1 /\left(6\left|k_{2}\right| \mu\right)}$, with $\frac{\partial \mathrm{ck}}{\partial u}=$ $-1 / \sqrt{3\left|k_{2}\right| \mu}$ and $\frac{\partial^{2} \mathrm{ck}}{\partial u^{2}}=-\infty$. Then, there is no regular periodic solution. This implies that the oscillatory parameter $\mu$ is restricted to the finite range

$$
0 \leq \mu \leq \mu_{\max } \quad \text { with } \quad \mu_{\max }=\frac{1}{6\left|k_{2}\right|} .
$$

It must also satisfy the upper bound (76), so that at each radius $\mu(x)$ is restricted to the range

$$
0 \leq \mu \leq \min \left(\mu_{+}(x), \mu_{\max }\right) .
$$

\section{B. Flux $F(\mu, x)$ as a function of $\mu$}

We show in Fig. 1 the flux $F(\mu, x)$ as a function of $\mu$ for several values of the radius $x$, for the case

$$
k_{2}=-\frac{1}{2}, \quad \mu_{\max }=\frac{1}{3} .
$$

A change of $\left|k_{2}\right|$ can be absorbed in a change of $\mu$ at constant $\left|k_{2}\right| \mu$, so this figure describes all Lagrangians with a quartic derivative self-interaction $X^{2}$.

The fixed upper bound (94), due to the fact that $G^{-1}(y)$ is not defined for all positive $y$, makes an important change to the generic behavior described above after Eq. (76) and seen to be universal at large radii in Sec. IV. At large radii we recover the single peak with a vanishing flux at both end points. The peak moves to lower $\mu$ with an increasing height as the radius $x$ increases, in agreement with Sec. IV. However, close to the Schwarzschild radius only part of the left side of the peak can be reached as (94) prevents access to larger values of $\mu$ and in particular the peak itself. This implies that a steady solution with constant flux $F$ can only exist for low values of the flux, $F \leq F\left(\mu_{\max }, 1 / 4\right)$, below the maximal flux reached at the Schwarzschild radius, $x=1 / 4$. Moreover, starting from the horizon we can see that a regular solution can only follow the left branch $\mu_{1}(x)$, on the left side of the peak. This is inconsistent with the large-radius boundary condition (89), which requires being on the right branch $\mu_{2}$ at large radii. Therefore, in contrast with the case of a quartic potential investigated in [33], there is no continuous and steady global solution that applies down to the $\mathrm{BH}$ horizon while converging to the static soliton at large radii. This means that, contrary to the quartic potential, the quartic derivative self-interaction $-X^{2}$ is not able to support the scalar cloud against the $\mathrm{BH}$ gravity in the relativistic regime. Then, we expect the supermassive BH to "eat" the scalar cloud in a short time, as compared with the age of the Universe.

\section{Constant flux solution}

We study in more detail the continuous solutions defined by a constant flux $F$. Because these solutions no longer connect to the soliton, the parameter $\alpha$ introduced in Eq. (34), understood as the definition of the common angular frequency $\omega_{0}$ in units of $m$, is no longer related to the value $\alpha$ associated with the soliton in (31). In particular, we no longer have the relation (28) between the gravitational and self-interaction potentials. Indeed, we no longer have hydrostatic equilibrium at large radii.

Once we have chosen a value $F$ for the flux, which is below the maximum value reached at the horizon $F_{\max }(x=1 / 4) \simeq 0.1 F_{s}$, the function $\mu(r)$ is set at all radii by the intersection of the left side of the peak of $F(\mu, x)$ with $F$; see Fig. 1 . From $\mu(r)$ we obtain the amplitude $\phi_{0}(r)$ from Eq. (50), while $\beta^{\prime}$ is given by Eq. (49). This fully determines $\beta(r)$, up to an irrelevant integration constant, and the scalar field (32) at radii $r \ll r_{\text {sg }}$, where the metric is dominated by the $\mathrm{BH}$ and given by Eqs. (10) and (11) or (15). At larger radii, the metric would be set by 
the scalar cloud self-gravity and we would solve in a selfconsistent manner the Poisson equation.

For $\mu \ll 1$ the scalar field reduces to the cosine (77) at lowest order, $\operatorname{as} \operatorname{ck}(u, 0) \simeq \cos (u)$, and the kinetic factor $X$ from Eq. (43) reads

$X=\mu \sin ^{2}\left(\omega_{0} t-\pi \beta / 2\right)=\frac{m^{2} \phi_{0}^{2}}{2 \Lambda^{4}} \sin ^{2}\left(\omega_{0} t-\pi \beta / 2\right)$,

where we used Eq. (50). In this small- $X$ regime we also have $K \simeq X, K^{\prime} \simeq 1$, and the scalar-field energy density reads at lowest order

$$
r \gg r_{s}: \rho_{\phi}=\frac{m^{2} \phi_{0}^{2}}{2}=\mu \Lambda^{4} .
$$

We recover the nonrelativistic regime, as in Eq. (31), that applied to the nonrelativistic soliton. Using Eqs. (81) and (72) this also reads as

$$
r \gg r_{s}: \rho_{\phi}=\frac{|F|}{r^{2} \sqrt{2(\alpha-\Phi)}}=\frac{F}{r^{2} v_{r}},
$$

where in the second equality we used Eq. (84). We recover the nonrelativistic definition of the flux, $F=r^{2} \rho_{\phi} v_{r}$.

The gravitational potential becomes dominated by the scalar cloud gravity when the mass $M_{\phi}$ associated with the scalar field within the radius $r$ is of the order of the black hole mass. Writing $M_{\phi} \sim \rho_{\phi} r^{3}$ and $\alpha-\Phi \sim-\Phi \sim r_{s} / r$, as $\Phi \sim \Phi_{\mathrm{BH}}$ up to this transition radius, we obtain

$$
r_{\mathrm{sg}} \sim r_{a}\left(\frac{F}{F_{s}}\right)^{-2 / 3}\left(\frac{r_{a}}{r_{s}}\right)^{1 / 3} .
$$

As $F / F_{s}<1$ from Fig. 1 and $r_{a} \gg r_{s}$, where $r_{a}$ is the characteristic radius defined in Eq. (26), we obtain for the transition radius $r_{\mathrm{sg}} \gg r_{a}$. This corresponds to a radius that is much larger than the soliton radius $R_{\mathrm{sol}}=\pi r_{a}$, recalled below Eq. (26). Therefore, we conclude that for these continuous solutions all radii are dominated by the $\mathrm{BH}$ gravity. This is because the high infall velocity, $\left|v_{r}\right| \sim \sqrt{-\Phi}$, implies a much smaller density, $\rho_{\phi}=F /\left(r^{2} v_{r}\right)$, than for the second branch $\mu_{2}(r)$ that converges to the static soliton, associated with the much smaller velocity (85). Thus, these solutions describe the latest stages of the infall of the scalar cloud onto the central $\mathrm{BH}$, when most of the scalar-field cloud has already been eaten by the $\mathrm{BH}$.

\section{Free-fall flux}

Another interpretation of the result (100) can be obtained from the flux expected in the case of free fall. At large radii, $r \gg r_{s}$, the scalar field is in the nonrelativistic regime and we expect the scalar-field cloud to fall into the central $\mathrm{BH}$ with the free-fall velocity $v_{r} \sim-\sqrt{2 \mathcal{G M} / r}$. Using
$F=r^{2} \rho_{\phi} v_{r}$ in the nonrelativistic regime and the definition of $F_{s}$ in Eq. (72), we obtain

$$
\frac{F}{F_{s}} \sim\left(\frac{M}{M_{\mathrm{BH}}}\right)^{2}\left(\frac{r}{r_{a}}\right)^{-3}\left(\frac{\rho}{\bar{\rho}_{c}}\right)^{-1 / 2} \frac{1}{H r_{a}},
$$

where $\bar{\rho}_{c}$ is the cosmological critical density and $H$ the Hubble expansion rate. For $r \sim r_{a}$, with $r_{a} \sim 20 \mathrm{kpc}, \rho \sim$ $10^{6} \bar{\rho}_{c}$ and $M \sim 10^{3} M_{\mathrm{BH}}$, we obtain $F / F_{s} \sim 10^{8}$. Such a large flux cannot be accommodated by the solutions shown in Fig. 1. This explains why we found in Eq. (100) that the continuous solution with the profile (99) can only describe at best the late stages of the infall, after most of the scalar mass has disappeared into the $\mathrm{BH}$ and only a small scalar mass remains, which can be transported with a small flux. Therefore, the infall of the scalar cloud cannot be described by the oscillatory solutions (32).

In any case, the fact that there are no regular solutions that satisfy both boundary conditions, at the horizon and at the soliton core, shows that such scalar-field models cannot support a stable galactic-mass scalar cloud around a supermassive BH. Therefore, they cannot provide realistic darkmatter scenarios. This shows the importance of checking the self-consistency of the system from the galactic kpc scales down to the Schwarzschild radius and taking into account the relativistic regime. Indeed, as recalled above, at large radii in the nonrelativistic regime this model is equivalent to a quartic potential model and the derivative self-interaction $-X^{2}$ builds an effective pressure that is able to support the scalar cloud against gravity. It happens that close to the Schwarzschild radius one enters the nonlinear regime, where the $X^{2}$ term is no longer a small correction to the standard kinetic term, and there the scalar field is no longer able to provide a self-consistent support against the $\mathrm{BH}$ gravity.

At large $X$ the sign of the kinetic term also becomes negative, $K^{\prime}<0$, which typically signals the appearance of ghosts. Thus, such a theory is also problematic at a more fundamental theoretical level. Hence, we do not consider this theory further.

\section{CONDITIONS TO STABILIZE THE SOLITON}

We have seen in the previous section that when the function $G^{-1}(y)$ is not defined over all positive $y$, as happened for the quartic Lagrangian with $k_{2}<0$, it may be impossible to obtain a steady state solution that satisfies both small and large radii boundary conditions. We investigate in this section the conditions to obtain global solutions that can match the static soliton at large radii. For simplicity, we focus on the large- $X$ behavior, where we assume that the kinetic function $K(X)$ behaves as a powerlaw (with $K^{\prime}>0$ ),

$$
X \gg 1: K(X) \simeq a X^{\nu}, \quad a>0, \quad \nu>0 .
$$


This yields for the functions $G(X)$ and $G^{-1}(y)$

$$
G(X)=a(2 \nu-1) X^{\nu}, \quad G^{-1}(y)=\left[\frac{y}{a(2 \nu-1)}\right]^{1 / \nu},
$$

with the constraint

$$
\nu>\frac{1}{2},
$$

so that $G(X)$ is monotonically increasing and $G^{-1}$ is well defined. Then, the quarter of period $\mathbf{Q}$ of Eq. (60) reads

$\mathbf{Q}=\mu^{(\nu-1) /(2 \nu)}[a(2 \nu-1)]^{1 /(2 \nu)} \frac{\sqrt{\pi} \Gamma[1-1 /(2 \nu)]}{2 \Gamma[3 / 2-1 /(2 \nu)]}$.

As explained in Sec. III C, the flux $F(\mu, x)$ as a function of $\mu$ generically shows a peak and vanishes at both end points of the range (76). The large-radius boundary condition selects the low-velocity branch (82) associated with the right side of the peak, while the small-radius boundary condition selects the high-velocity branch (81) associated with the left side of the peak. To find a global solution that smoothly switches from the left to the right branch, the height $F_{\text {peak }}$ of the peak must increase at both large and small radii. We have seen that this is always the case at large radii, in the nonrelativistic regime (80). At small radii, this depends on the large- $X$ behavior of the kinetic function, and we have seen in Sec. V that for the quartic Lagrangian this does not happen, as the peak is cut from the right by the additional upper bound (94). Then, although this is neither a sufficient nor a necessary condition, models that are likely to show the desired behavior for $F(\mu, x)$ should show the full peak, with the vanishing of the flux at both end points 0 and $\mu_{+}$. At the horizon the metric function $f(r)$ vanishes. From Eq. (76) this means that in order to reach $\mu_{+}(x)$, down to the horizon, the quarter of period $\mathbf{Q}$ must decrease down to 0 for large $\mu$. In the powerlaw case (105) this gives the constraint

$$
\nu<1 \text {, }
$$

so that $\mathbf{Q}$ vanishes for $\mu \rightarrow \infty$. From Eq. (75) we obtain

$$
C_{\mu}=\frac{\nu}{3 \nu-1},
$$

and the flux (70) reads

$$
\begin{aligned}
\frac{F}{F_{s}}= & x^{2} h \frac{\nu[a(2 \nu-1)]^{1 / \nu} \Gamma[1-1 /(2 \nu)]^{2}}{(3 \nu-1) \pi \Gamma[3 / 2-1 /(2 \nu)]^{2}} \mu^{(2 \nu-1) / \nu} \\
& \times \sqrt{1-\frac{\pi \Gamma[3 / 2-1 /(2 \nu)]^{2} f \mu^{(1-\nu) / \nu}}{(1+\alpha)^{2}[a(2 \nu-1)]^{1 / \nu} \Gamma[1-1 /(2 \nu)]^{2}}} .
\end{aligned}
$$

At the horizon $x$ and $h$ are finite while $f$ vanishes. We can see that $\mu_{+}$grows as $f^{-\nu /(1-\nu)}$ and the peak height grows as $f^{-(2 \nu-1) /(1-\nu)}$. Therefore, we find indeed the required behavior for a global transonic solution, as the peak of $F(\mu)$ is fully obtained at small radii with a height that grows as we move closer to the horizon.

As we explicitly note in Sec. VII C, the condition $1 / 2<$ $\nu<1$ that we have obtained here is only suggestive. It is neither a necessary nor a sufficient condition. For instance, kinetic functions $K(X)$ that show the asymptotic slope $K(X) \sim X^{\nu}$ with $1 / 2<\nu<1$ but are badly behaved for intermediate values of $X$, e.g., if they violate the condition (58) at intermediate $X$, cannot provide a realistic or physical model. On the other hand, we see in Sec. VII, on the example (109) of a well-behaved kinetic function $K(X)$, that $X$ actually remains bounded down to the BH horizon, $0 \leq X \leq X_{\max }(x=1 / 4)$. Then, the very large $X$ behavior of $K(X)$, at values that are not reached in practice, is actually irrelevant. It may however be probed by other configurations, e.g., in the early Universe.

\section{EXPLICIT EXAMPLE}

\section{A. Characteristic functions}

To illustrate the results of the previous section we consider the case

$$
K(X)=(1+3 X / 2)^{2 / 3}-1 .
$$

This corresponds to the exponent $\nu=2 / 3$, which falls in the range $1 / 2<\nu<1$ obtained in Eqs. (104) and (106). This also gives the quadratic coefficient $k_{2}=-1 / 2$, as for the quartic model (96),

$$
\nu=2 / 3, \quad k_{2}=-1 / 2 .
$$

The function $G(X)$ reads as

$G(X)=\frac{1}{3}(1+3 X / 2)^{2 / 3}-\frac{4}{3}(1+3 X / 2)^{-1 / 3}+1$,

and the inverse function $G^{-1}(y)$ as

$G^{-1}(y)=\frac{2}{3}\left[\frac{\left(-1+y+\left(2+\sqrt{5-3 y+3 y^{2}-y^{3}}\right)^{2 / 3}\right)^{3}}{2+\sqrt{5-3 y+3 y^{2}-y^{3}}}-1\right]$.

At large $y$ this gives the power-law asymptote

$$
y \rightarrow \infty: G^{-1}(y)=2 \sqrt{3} y^{3 / 2}+\ldots
$$

The nonlinear differential equation (53) now admits regular periodic solutions $\operatorname{ck}(u, \mu)$ for all positive $\mu$. Then, at any radius $x$ the oscillatory parameter $\mu$ is only bounded by $\mu_{+}(x)$ from Eq. (76). 


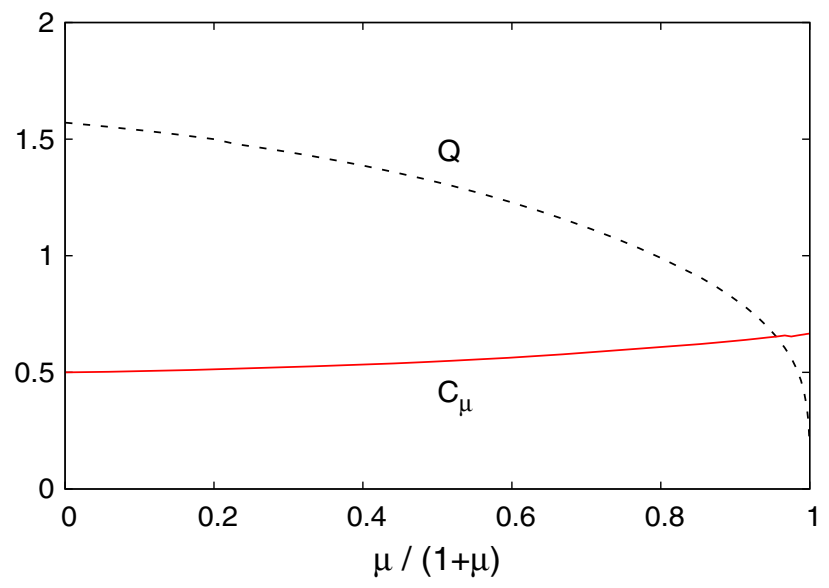

FIG. 2. Quarter of period $\mathbf{Q}$, from Eq. (60), and average $C_{\mu}$, from Eq. (75), as functions of the oscillatory parameter $\mu$. We use the ratio $\mu /(1+\mu)$ for the abscissa, to cover the range $0 \leq \mu<\infty$.

We show in Fig. 2 the quarter of period $\mathbf{Q}$ and the average $C_{\mu}$ as functions of $\mu$. In agreement with the analysis of Sec. VI, $\mathbf{Q}$ goes to 0 as $\mu$ goes to infinity, while $C_{\mu}$ remains finite and does not vary much over the full range $0 \leq \mu<\infty$. More precisely, we have the asymptotic behaviors

$$
\mu \rightarrow \infty: \mathbf{Q}=\frac{\sqrt{2 \pi} \Gamma[5 / 4]}{3^{1 / 4} \Gamma[3 / 4]} \mu^{-1 / 4}, \quad C_{\mu}=\frac{2}{3} .
$$

We find in Sec. VII B that $\mu$ grows at smaller radii and reaches at the horizon the value $\mu_{s} \simeq 3.5$ of Eq. (116) below. This corresponds to $\mathbf{Q}_{s} \simeq 1$ and $C_{\mu_{s}} \simeq 0.6$. Therefore, the oscillatory parameter $\mu$ reaches the mildly nonlinear regime at the Schwarzschild radius. There, all higher-order terms of the kinetic function $K(X)$ are relevant, as is obvious from the fact that the physics associated with the kinetic function (109) is quite different from the one associated with the quartic case analyzed in Sec. V (a quasistatic soliton can now be supported around the supermassive BH).

We display in Fig. 3 the oscillatory function $\mathrm{ck}(u, \mu)$ for $\mu=0$, where $\operatorname{ck}(u, 0)=\cos (u)$, and for $\mu=\mu_{s} \simeq 3.5$. We only show the first period, $0 \leq u \leq 4 \mathbf{Q}$, and we renormalize the abscissa by $\mathbf{Q}$. We can see that although $\mathbf{Q}$ has decreased from $\mathbf{Q}(0)=\pi / 2$ to $\mathbf{Q}_{s} \simeq 1$, the shape of the function $\operatorname{ck}(u)$ remains close to the cosine once we renormalize the period.

\section{B. Critical solution}

We show in Fig. 4 the flux $F(\mu, x)$ as a function of $\mu$ for several values of the radius $x$. Close to the horizon the peak moves to large values of $\mu$, as $\mu_{+} \rightarrow \infty$ for $x \rightarrow 1 / 4$. To display the curves from $x=50$ to $x=0.4$ on the same plot we use the abscissa $\mu /(1+\mu)$ in Fig. 4. In agreement with the analysis of the previous section, we now find that

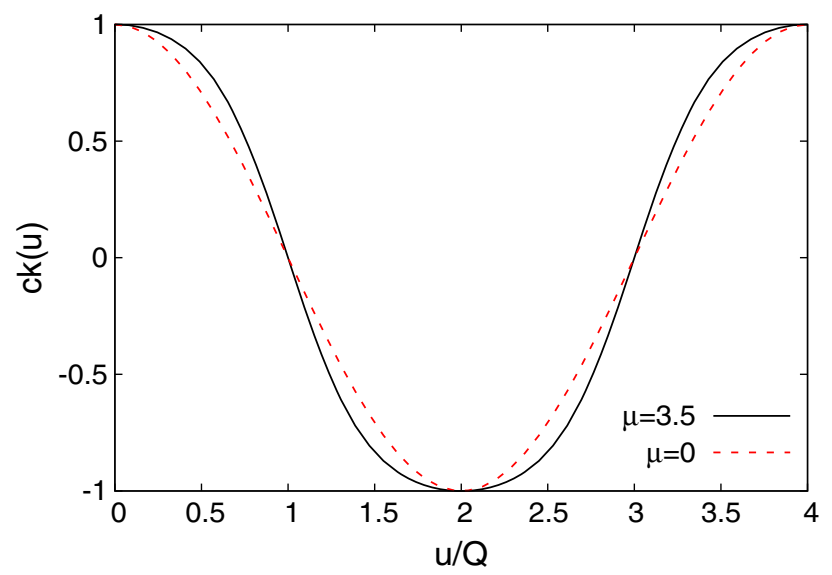

FIG. 3. Nonlinear oscillatory function $\operatorname{ck}(u, \mu)$, for $\mu=3.5$ (solid line) and $\mu=0$ (dashed line). The abscissa is renormalized by the quarter of period $\mathbf{Q}$.

$F(\mu)$ shows a full peak, with a vanishing flux at both end points, at any radius $x$. Moreover, the peak height increases for both large and small radii, with a minimum $\left|F_{c}\right|=$ $F_{\star}\left|F_{s}\right|$ at the intermediate radius $x_{\star}$,

$$
\frac{F_{c}}{F_{s}}=F_{\star} \quad \text { with } \quad x_{\star} \simeq 1.4, \quad F_{\star} \simeq 0.9
$$

We show the curve $F_{\text {peak }}(x) / F_{s}$ of the peak height, as a function of the radius $x$, in Fig. 5. This clearly shows the increase of the peak height at small and large radii and the minimum at $x_{\star}$.

A global solution is obtained provided the constant flux $|F|$ is smaller than the critical value $\left|F_{c}\right|$, so that at any radius $x$ above the horizon there is at least one solution $\mu(x)$

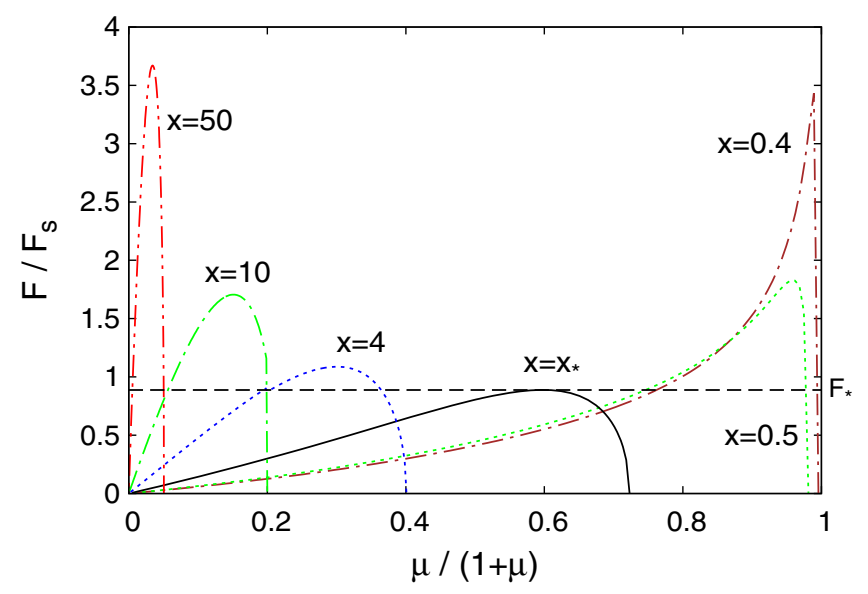

FIG. 4. Normalized flux $F(\mu, x) / F_{s}$ as a function of the oscillatory parameter $\mu$, for various values of the radial coordinate $x$, from Eq. (70) for the kinetic function (109). We use the ratio $\mu /(1+\mu)$ for the abscissa, so that large values of $\mu$ fit into the figure. The horizontal dashed line is the value $F_{\star}$ of Eq. (115), defined as the minimum over all radii, $1 / 4 \leq x<\infty$, of the height of the peak. It is reached at radius $x_{\star} \simeq 1.4$. 


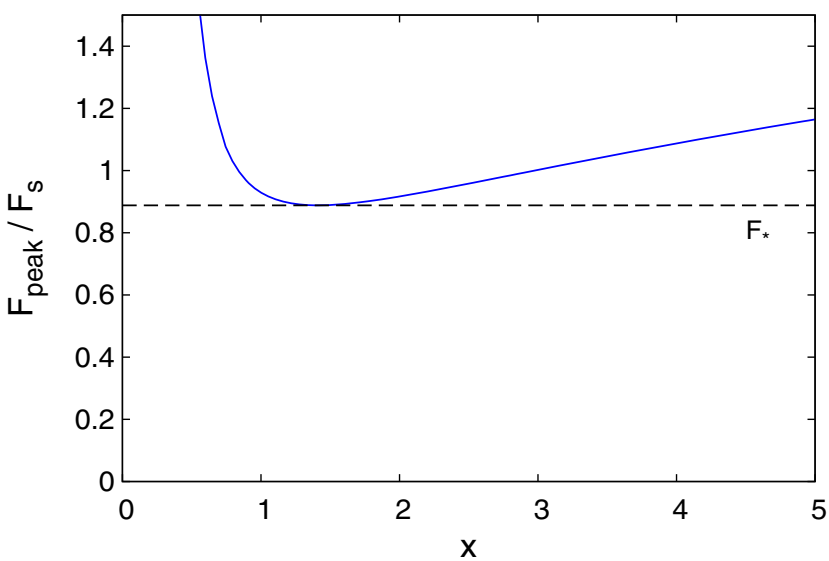

FIG. 5. Peak value $F_{\text {peak }}(x) / F_{s}$ as a function of the radial coordinate $x$. The horizontal dashed line is the minimum value $F_{\star} \simeq 0.9$, reached at $x_{\star} \simeq 1.4$.

to Eq. (70), understood as an implicit equation for $\mu$. For $|F|<\left|F_{c}\right|$ there are two solutions $\mu_{1}<\mu_{2}$ at any radius and we recover the high- and low-velocity branches discussed below (76) and analyzed in the limit of large radii in Sec. IV A. We have seen in (90) that at small radii, near the horizon, we must follow the left branch $\mu_{1}(x)$, associated with large infall velocities. On the other hand, we have seen in (89) that at large radii we must follow the right branch $\mu_{2}$, associated with small infall velocities, to converge to the static soliton. These two boundary conditions select the critical value $F_{c}$ as the only physical value for the flux, which allows us to obtain a regular solution $\mu_{c}(x)$ that smoothly connects the two branches at radius $x_{\star}$, where they meet.

We show the two branches $\left\{\mu_{1}(x), \mu_{2}(x)\right\}$ in Fig. 6 for the critical flux $F_{c}$ and for a lower flux $F_{c} / 3$. In the case $F_{c} / 3$, these two branches remain well separated at all radii and we cannot switch from one side of the peak to the other.

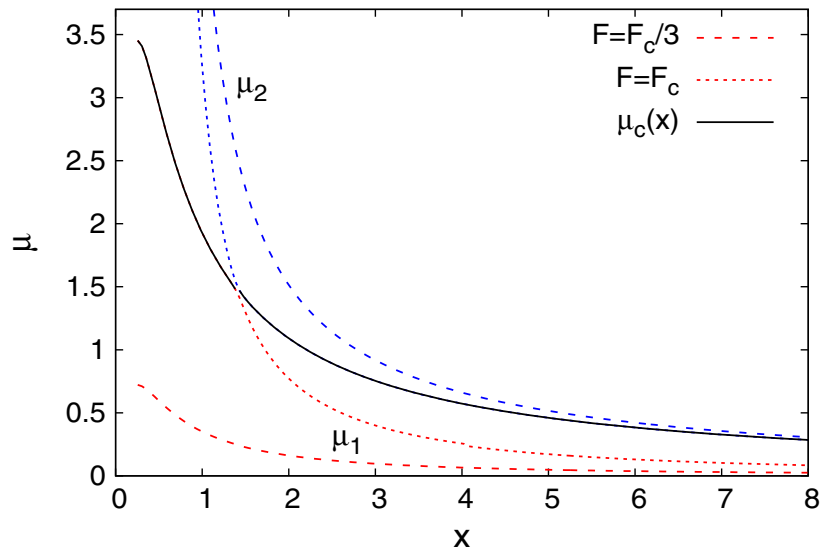

FIG. 6. Oscillatory parameters $\mu_{1}(x)$ and $\mu_{2}(x)$ for a constant flux $F_{c} / 3$ (dashed lines) and $F_{c}$ (dotted lines). The critical curve $\mu_{c}(x)$ (solid line) is equal to $\mu_{1}$ for $x<x_{\star}$ and to $\mu_{2}$ for $x>x_{\star}$, with $F=F_{c}$.
Only for the critical value $F_{c}$ can we switch from $\mu_{1}$ to $\mu_{2}$ in a continuous manner, as shown in the figure. This is similar to the selection of the unique transonic solution in the hydrodynamical case [37]. At large radii, in the weakgravity regime, $\mu_{c}(x)=\mu_{2}(x)$ is given by Eq. (82). Close to the horizon $\mu_{c}(x)=\mu_{1}(x)$ remains finite and our numerical computation shown in Fig. 6 gives

$$
r \rightarrow r_{s} / 4: \mu_{c}(x) \rightarrow \mu_{s} \text { with } \quad \mu_{s} \simeq 3.5 \text {. }
$$

The fact that $\mu$ is of order unity near the horizon shows that the scalar field is in the nonlinear regime, where all nonlinear terms in the kinetic function $K(X)$ are relevant.

\section{Radial profile}

Thus, the scalar field $\phi(r, t)$ depends on both radius and time as determined by the solution (32), where the parameter $\mu$ of the nonlinear oscillator $\operatorname{ck}(u, \mu)$ follows the critical solution $\mu_{c}(x)$ displayed in Fig. 6 above. At each radius, the kinetic argument $X$ oscillates with time, as seen in Eq. (43). Using Eq. (57), this also reads as $X=G^{-1}\left[\mu\left(1-\mathrm{ck}^{2}\right)\right]$, so that $X$ oscillates in the range

$$
0 \leq X(r, t) \leq X_{\max }(r) \quad \text { with } \quad X_{\max }=G^{-1}(\mu) .
$$

We show again in Fig. 7 the radial profile of the oscillatory parameter $\mu(x)$, given by the critical curve $\mu_{c}(x)$ of Fig. 6, as well as the upper value $X_{\max }(x)$. We can see that $X_{\max } \simeq$ 16.5 and $K^{\prime}\left(X_{\max }\right) \simeq 0.33$ at the horizon. Although we reach the nonlinear regime, $K^{\prime}$ is still of order unity. Because $X$ remains finite at the horizon, the function $K(X)$ can deviate from the expression (109) for $X>$ $X_{\max }\left(r_{s} / 4\right)$ without changing our results. This implies that the function $K(X)$ is not required to show the power-law behavior $K \sim X^{2 / 3}$ at infinity and a broader class of kinetic functions are able to support the scalar-field soliton around the supermassive $\mathrm{BH}$.

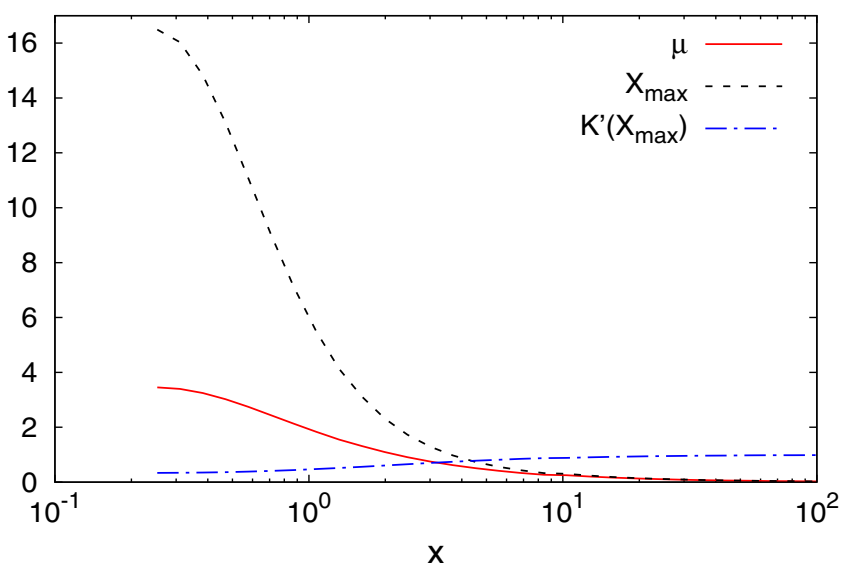

FIG. 7. Radial profile of the oscillatory parameter $\mu(x)$, of the upper bound $X_{\max }(x)$ of Eq. (117), and of the first derivative $K^{\prime}\left[X_{\max }(x)\right]$. 


\section{Behavior at the Schwarzschild radius}

\section{Isotropic coordinates}

As for the case of the quartic potential [33], the effective velocity $v_{r}=\pi \beta^{\prime} /(2 m)$ diverges at the Schwarzschild radius because of the metric factor $1 / f$. Thus, from Eq. (52) we obtain close to the Schwarzschild radius, where $f \rightarrow 0$,

$$
\begin{aligned}
r \rightarrow r_{s} / 4: \frac{\pi \beta^{\prime}}{2 m} & \sim-(1+\alpha) \sqrt{\frac{h}{f}} \sim-\frac{(1+\alpha) 8 r_{s}}{4 r-r_{s}}, \\
\beta & \sim-\frac{(1+\alpha) 4 m r_{s}}{\pi} \ln \left(\frac{4 r-r_{s}}{4 r_{s}}\right) .
\end{aligned}
$$

However, this divergence is only an artefact, due to the use of the isotropic coordinates (9).

\section{Eddington coordinates}

As in [33], to check that the scalar field remains well behaved down to the horizon it is convenient to introduce the Eddington coordinates $(\tilde{t}, \tilde{r})$, where $\tilde{r}$ is the standard Schwarzschild radial coordinate of Eq. (13) and $\tilde{t}$ is the Eddington time, defined by [35]

$$
\tilde{t}=t+r_{s} \ln \left|\frac{\tilde{r}}{r_{s}}-1\right| .
$$

This gives the metric

$d s^{2}=-\left(1-\frac{r_{s}}{\tilde{r}}\right) d \tilde{t}^{2}+2 \frac{r_{s}}{\tilde{r}} d \tilde{t} d \tilde{r}+\left(1+\frac{r_{s}}{\tilde{r}}\right) d \tilde{r}^{2}+\tilde{r}^{2} d \vec{\Omega}^{2}$,

which is regular over all $\tilde{r}>0$. These coordinates $(\tilde{t}, \tilde{r})$ are directly related to the Eddington-Finkelstein coordinates [35].

Substituting the result (118) into Eq. (32) gives

$$
\tilde{r} \rightarrow r_{s}: \phi=\phi_{s} \operatorname{ck}\left[\frac{2 \mathbf{Q}_{s}}{\pi}(1+\alpha) m(\tilde{t}+\tilde{r}), \mu_{s}\right],
$$

where the parameter $\mu_{s}$ at the Schwarzschild radius was obtained in Eq. (116) and the amplitude $\phi_{s}$ is given by Eq. (50) in terms of $\mu_{s}$. As for the free scalar and for the case of a quartic potential [33], the scalar field is well defined at the horizon and we recover a purely ingoing solution with unit velocity. Nevertheless, the derivative self-interactions remain relevant down to the horizon as (121) differs from the cosine (i.e., harmonic) expression of the free case. We obtain a nonlinear radial wave, with higher-order harmonics given by the expansion (62).

We can now come back to the definition of the velocity. In the large-radius limit, we have identified $v_{r}=\pi \beta^{\prime} /(2 m)$ of Eq. (52) with the velocity obtained in the fluid picture of the nonrelativistic dark matter through the Euler equation (23) and Eq. (21). In fact, Eq. (49) allows us to go beyond this large-radius regime. Indeed, we can identify this relation with the relativistic dispersion relation of a particle of mass $m$ and momentum $p^{\mu}, g_{\mu \nu} p^{\mu} p^{\nu}=-m^{2}$, with

$$
p^{0}=\frac{2 \mathbf{Q} \omega_{0}}{\pi f}, \quad p^{r}=\frac{\mathbf{Q} \beta^{\prime}}{h} .
$$

Then the speed can be identified as

$$
c^{r}=\frac{p^{r}}{p^{0}}=\frac{f}{h} \frac{\pi \beta^{\prime}}{2 \omega_{0}},
$$

which coincides with $v_{r}$ in the large-radius limit and for $\alpha \ll 1$. Close to the BH horizon, we have seen that Eq. (50) gives

$$
r \rightarrow r_{s} / 4: \beta^{\prime} \simeq-\frac{2 \omega_{0}}{\pi} \sqrt{\frac{h}{f}}
$$

as $f \rightarrow 0$ while $\mathbf{Q}$ remains finite; see also Eq. (118). This yields

$$
r \rightarrow r_{s} / 4: c^{r} \simeq-\sqrt{\frac{f}{h}} .
$$

If we use the Schwarzschild radial coordinate $\tilde{r}$ instead of the isotropic radial coordinate $r$, we have from Eq. (13) $d \tilde{r} / d r=\sqrt{f h}$ and we obtain

$$
c^{\tilde{r}}=\sqrt{\frac{f^{3}}{h}} \frac{\pi \beta^{\prime}}{2 \omega_{0}},
$$

and

$$
\tilde{r} \rightarrow r_{s}: c^{\tilde{r}} \simeq-f .
$$

We find that both velocities vanish at the horizon. We recover the well-known result that the velocity of infalling matter measured by a distant observer (at rest at infinity, with a proper time given by $t$ ) vanishes as the body approaches the horizon. On the other, the proper time of a particle that is falling from infinity at rest is $d \tau=f d t$ [38]. Thus, we recover $d \tilde{r} / d \tau=-1$ at the horizon following the infalling matter. The dynamics become highly relativistic as we approach the horizon.

\section{E. Density profile}

For spherically symmetric configurations, the density defined by the time-time component of the energy-momentum tensor in the coordinates $(\tilde{t}, \tilde{r})$ reads 


$$
\begin{aligned}
\tilde{\rho}_{\phi} \equiv & -\tilde{T}_{0}^{0}=(2-f) K^{\prime}\left(\frac{\partial \phi}{\partial \tilde{t}}\right)^{2}+(f-1) K^{\prime} \frac{\partial \phi}{\partial \tilde{t}} \frac{\partial \phi}{\partial \tilde{r}} \\
& -\Lambda^{4} K+\frac{m^{2}}{2} \phi^{2},
\end{aligned}
$$

and the partial derivatives are related by

$$
\frac{\partial \phi}{\partial \tilde{t}}=\frac{\partial \phi}{\partial t}, \quad \frac{\partial \phi}{\partial \tilde{r}}=\frac{\partial \phi}{\partial r} \frac{1}{\sqrt{f h}}+\frac{\partial \phi}{\partial t}\left(1-\frac{1}{f}\right) .
$$

For the solution (32), with Eqs. (36) and (38), this gives

$$
\begin{aligned}
\frac{\tilde{\rho}_{\phi}}{\Lambda^{4}}= & \mu \mathrm{ck}^{2}-K+K^{\prime}\left(\frac{\partial \mathrm{ck}}{\partial u}\right)^{2} 2 \mu\left[\frac{2 \mathbf{Q}}{\pi}(1+\alpha)\right]^{2} \\
& \times\left\{\frac{1}{f}+\frac{f-1}{f} \sqrt{1-\frac{\pi^{2} f}{(1+\alpha)^{2} 4 \mathbf{Q}^{2}}}\right\} .
\end{aligned}
$$

This energy density remains finite at the Schwarzschild radius. Neglecting $\alpha \ll 1$, we obtain

$$
\tilde{r}=r_{s}, \quad r=\frac{r_{s}}{4}:\left\langle\tilde{\rho}_{\phi}\right\rangle \simeq 3.2 \Lambda^{4} \simeq 0.6 \rho_{a},
$$

where $\langle\ldots\rangle$ denotes the average over the fast oscillations over time, as in Eqs. (73)-(75). Contrary to the case of the free scalar, where the flux $F$ and the density $\tilde{\rho}_{\phi}$ can take any value, for the self-interacting scalar field $F$ and $\tilde{\rho}_{\phi}$ are uniquely determined (because the system becomes nonlinear). As could be expected, the density (131) is set by the characteristic density $\rho_{a}$ defined in Eq. (24), which measures the strength of the self-interactions. The unboundedness of the free case is recovered by the fact that $\left\langle\tilde{\rho}_{\phi}\right\rangle \rightarrow \infty$ when $\rho_{a} \rightarrow \infty$, which corresponds to vanishing self-interactions, $k_{2} \rightarrow 0$.

In the weak-gravity regime, dominated by the $\mathrm{BH}, \mu(x)$ follows the low-velocity branch $\mu_{2}(x)$ of Eq. (82). This gives

$$
r_{s} \ll r \ll r_{\mathrm{sg}}: \mu \simeq-\frac{16 \Phi}{3} \simeq \frac{8 r_{s}}{3 r},
$$

where we used Eq. (15). In this regime $\mu \ll 1$ and at leading order the density (130) gives

$$
r_{s} \ll r \ll r_{\mathrm{sg}}: \frac{\left\langle\tilde{\rho}_{\phi}\right\rangle}{\Lambda^{4}} \simeq \mu \simeq \frac{8 r_{s}}{3 r} \propto r^{-1}
$$

while the velocity (85) reads

$$
r_{s} \ll r \ll r_{\mathrm{sg}}: v_{r} \simeq-\frac{3 F_{\star}}{4} \frac{r_{s}}{r} \propto r^{-1} .
$$

As for the case of a quartic potential, the density decreases with radius as $1 / r$, more slowly than the $r^{-3 / 2}$ falloff

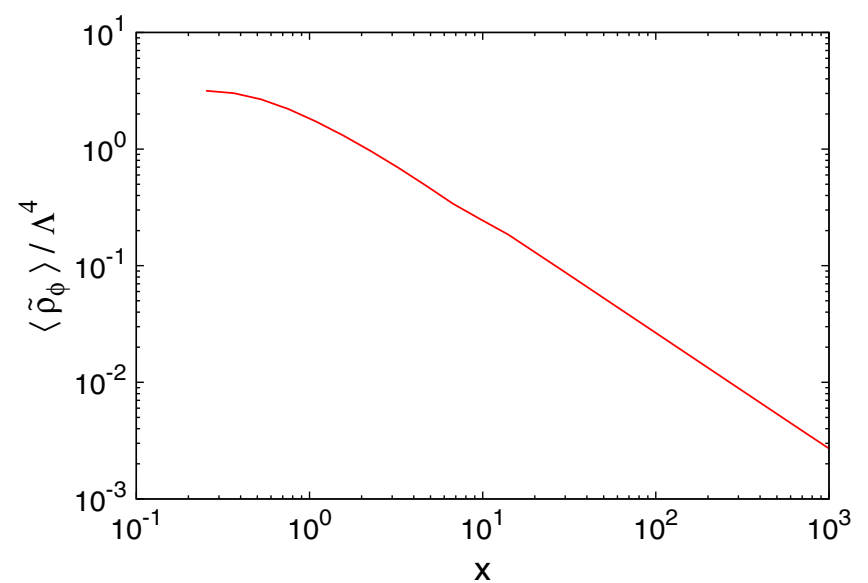

FIG. 8. Scalar-field energy density computed in the Eddington metric, from the Schwarzschild radius up to $10^{4} r_{s}$, where the metric potentials are still dominated by the central $\mathrm{BH}$. We plot the average $\left\langle\tilde{\rho}_{\phi}\right\rangle$ in the fast oscillations with time.

obtained for the free scalar [33]. This is because the velocity decreases faster, as $1 / r$ instead of $1 / \sqrt{r}$, as the self-interactions give rise to an effective pressure that stabilizes the scalar cloud and enables the convergence to the static soliton solution at large radii. We show in Fig. 8 the scalar-field profile obtained from Eq. (130), averaged over the fast oscillations. It clearly displays the $1 / r$ profile (133) at large radii and the finite value (131) at the horizon.

\section{F. Lifetime of the scalar-field soliton}

At large radii, in the weak-gravity regime dominated by the scalar cloud self-gravity, the velocity (85) reads

$$
r_{\mathrm{sg}} \ll r \lesssim R_{\mathrm{sol}}: v_{r} \simeq-\frac{3 F_{\star}}{8} \frac{\rho_{a}}{\rho_{\mathrm{sol}}} \frac{r_{s}^{2}}{r^{2}},
$$

where we used Eqs. (28) and (24). This coincides with the result obtained in [33] for the case of a quartic potential. Indeed, as recalled in (7), in the nonrelativistic small-field regime the derivative self-interaction is equivalent to a potential self-interaction. Again, at radii of the order of the soliton radius $R_{\mathrm{sol}}=\pi r_{a}$, this gives the typical radial velocity $v_{r}$ and evolution timescale $t_{c}$,

$$
v_{r}\left(r_{a}\right) \sim-\frac{\rho_{a}}{\rho_{\mathrm{sol}}} \frac{r_{s}^{2}}{r_{a}^{2}}, \quad t_{c} \equiv \frac{r_{a}}{\left|v_{r}\right|} \sim r_{a} \frac{\rho_{\mathrm{sol}}}{\rho_{a}} \frac{r_{a}^{2}}{r_{s}^{2}} .
$$

This also reads

$$
t_{c} \sim t_{H}\left(\frac{\bar{\rho}_{c}}{\rho_{a}}\right)^{5 / 2} \frac{\rho_{\mathrm{sol}}}{\bar{\rho}_{c}}\left(\frac{R_{H}}{r_{s}}\right)^{2}
$$

where $t_{H}=1 / H$ and $R_{H}=1 / H$ are the Hubble time and Hubble radius, and $\bar{\rho}_{c}=3 H^{2} /(8 \pi \mathcal{G})$ is the cosmological critical density. At redshift $z=0$ this gives 


$$
t_{c} \sim 10^{3} t_{H} \frac{\rho_{\text {sol }}}{\bar{\rho}_{c}}\left(\frac{\rho_{a}}{1 \mathrm{eV}^{4}}\right)^{-5 / 2}\left(\frac{M}{10^{8} M_{\odot}}\right)^{-2}
$$

For the soliton to give rise to a significant departure from the CDM profiles on galactic scales, we must have a radius of about $20 \mathrm{kpc}$, which gives $\rho_{a} \sim 1 \mathrm{eV}^{4}$ [27]. Larger characteristic densities lead to smaller soliton radii. We typically have $\rho_{\text {sol }} / \bar{\rho}_{c} \sim 10^{5}$ for the DM overdensity in the soliton core. Therefore, we find that $t_{c} \gg t_{H}$ and the DM solitonic cores can easily survive until today, despite the infall of the inner layers into the central supermassive $\mathrm{BH}$.

Again, astrophysical stellar mass BHs cannot eat a significant fraction of the galactic DM soliton. Indeed, for $N$ BHs of unit solar mass, the typical timescale for the soliton depletion reads

$$
t_{N} \sim 10^{19} \frac{t_{H}}{N} \frac{\rho_{\mathrm{sol}}}{\bar{\rho}_{c}}\left(\frac{\rho_{a}}{1 \mathrm{eV}^{4}}\right)^{-5 / 2} .
$$

Since we typically have $N<10^{11}$, as only a fraction of the galactic baryonic mass can be made of stellar BHs, we obtain $t_{N} \gg 10^{8} t_{H}$ and the soliton mass loss is negligible.

\section{RENORMALIZATION OF THE ACTION}

We have seen in the previous sections that for nonlinear kinetic functions that satisfy conditions such as (104) and (106) the scalar field with the Lagrangian (1) displays wellbehaved solutions from the Newtonian to the relativistic regimes, i.e., from the small-field and weak-gravity regimes to the large-field and strong-gravity regimes. In this section, we check that quantum corrections remain small and do not invalidate the previous analysis, based on the classical equations of motion.

\section{A. Weak-gravity regime}

In the spirit of background quantization, we decompose the scalar field in the classical background $\bar{\phi}$, which is a solution of the classical equations of motion, and the quantum fluctuations $\hat{\phi}$,

$$
\phi=\bar{\phi}+\hat{\phi} .
$$

The kinetic argument also reads $X=\bar{X}+\hat{X}$, with

$$
\hat{X}=-\frac{1}{\Lambda^{4}} g^{\mu \nu} \partial_{\mu} \bar{\phi} \partial_{\nu} \hat{\phi}-\frac{1}{2 \Lambda^{4}} g^{\mu \nu} \partial_{\mu} \hat{\phi} \partial_{\nu} \hat{\phi}
$$

while the Lagrangian can be expanded as $\mathcal{L}=\overline{\mathcal{L}}+\hat{\mathcal{L}}$, with

$$
\hat{\mathcal{L}}=\Lambda^{4}\left(\bar{K}^{\prime} \hat{X}+\frac{\bar{K}^{\prime \prime}}{2} \hat{X}^{2}+\ldots\right)-\frac{m^{2}}{2}\left(2 \bar{\phi} \hat{\phi}+\hat{\phi}^{2}\right) .
$$

We first consider the weak-gravity regime, far from the $\mathrm{BH}$, where the background geometry is well described by the Minkowski spacetime and the background scalar field is scale independent,

$$
\bar{\phi}=\bar{\phi}(t), \quad \frac{d \bar{\phi}}{d t} \sim m \bar{\phi}
$$

This corresponds for instance to the core of the static soliton solution (31), where $\vec{v}=0$ and the phase $s$ only depends on time. Expanding the Lagrangian to second order in the perturbation, we get from Eq. (142) the secondorder variation

$\hat{\mathcal{L}}^{(2)}=\frac{\bar{K}^{\prime}+2 \bar{X} \bar{K}^{\prime \prime}}{2}\left(\frac{\partial \hat{\phi}}{\partial t}\right)^{2}-\frac{\bar{K}^{\prime}}{2}(\nabla \hat{\phi})^{2}-\frac{m^{2}}{2} \hat{\phi}^{2}$.

This can be the basis of a well-defined perturbation theory, without ghosts or small-scale instabilities in the linear regime, when we have

$$
\bar{K}^{\prime}>0, \quad \bar{K}^{\prime}+2 \bar{X} \bar{K}^{\prime \prime}>0 .
$$

We implicitly assumed $K^{\prime} \geq 0$ throughout this article. The second condition, $K^{\prime}+2 X K^{\prime \prime} \geq 0$, coincides with the condition (58) that was required to build the solution (32) from a well-defined nonlinear oscillatory function $\operatorname{ck}(u)$, as described in Sec. III B.

It is convenient to normalize the field $\hat{\phi}$ as

$$
\hat{\phi}=\frac{\varphi}{\sqrt{\bar{K}^{\prime}+2 \bar{X} \bar{K}^{\prime \prime}}} .
$$

After one integration by parts, this gives

$$
\hat{\mathcal{L}}^{(2)}=\frac{1}{2}\left(\frac{\partial \varphi}{\partial t}\right)^{2}-\frac{c_{s}^{2}}{2}(\nabla \varphi)^{2}-\frac{\bar{m}^{2}}{2} \varphi^{2},
$$

where the speed of sound $c_{s}$ is defined by

$$
c_{s}^{2}=\frac{\bar{K}^{\prime}}{\bar{K}^{\prime}+2 \bar{X} \bar{K}^{\prime \prime}}>0
$$

and the effective mass $\bar{m}$

$$
\bar{m}^{2}=\frac{m^{2}}{\bar{K}^{\prime}+2 \bar{X} \bar{K}^{\prime \prime}}-\frac{\frac{d^{2}}{d t^{2}}\left[\sqrt{\bar{K}^{\prime}+2 \bar{X} \bar{K}^{\prime \prime}}\right]}{\sqrt{\bar{K}^{\prime}+2 \bar{X} \bar{K}^{\prime \prime}}} .
$$

At low $\bar{X}$, that is, for small amplitude of the scalar field and $\bar{\rho}_{\phi} \ll \Lambda^{4}$, we have $\bar{K} \simeq \bar{X} \ll 1$ and

$$
\bar{X} \ll 1: c_{s}^{2} \simeq 1, \quad \bar{m} \simeq m .
$$

At large $\bar{X}$, for a power-law behavior $K(X) \sim X^{\nu}$ as in Eq. (102), we have 
$\bar{X} \gg 1: c_{s}^{2} \simeq \frac{1}{2 \nu-1}>0, \quad \bar{m} \sim \frac{m}{\sqrt{\bar{K}^{\prime}}} \gg m>0$,

provided the exponent $\nu$ verifies

$$
1 / 2<\nu<1
$$

For $\nu>1$ we still have $c_{s}^{2}>0$ but $\bar{K}^{\prime}$ becomes large and the squared mass becomes negative, as it is dominated by the second term in Eq. (149) which scales as $-(\nu-1)^{2} m^{2}$ for $d \bar{X} / d t \sim \pm m \bar{X}$. The bounds (152) coincide with the bounds (104) and (106) that were required at the classical level to obtain well-behaved global solutions, from the stronggravity to the weak-gravity regimes. Therefore, they are satisfied by realistic models, such as Eq. (109).

We are interested in quantum phenomena in the ultraviolet. In the infrared there are no divergences thanks to the scalar mass $m$, which is much larger than cosmological scales. Hence we neglect the time variation of $c_{s}$ and $\bar{m}$. From Eqs. (150) and (151) $c_{s}$ is always of order unity; therefore, we take $c_{s} \sim 1$ and omit factors $c_{s}$ in the order-ofmagnitude estimates below. Then, the propagator for the quantum field $\varphi$ behaves like

$$
G_{\varphi}(\omega, \vec{p})=\frac{1}{-\omega^{2}+\vec{p}^{2}+\bar{m}^{2}}=\frac{1}{p^{2}+\bar{m}^{2}} .
$$

Let us now consider the interaction terms. They spring from expressions like $\Lambda^{4} \bar{K}^{(n)} \frac{\hat{X}^{n}}{n !}$. In the following, we omit numerical factors and focus on the scalings with $\bar{X}$. Then, we write (141) as

$$
\hat{X} \sim \bar{X}^{1 / 2}\left(\frac{\partial \hat{\phi}}{\Lambda^{2}}\right)+\left(\frac{\partial \hat{\phi}}{\Lambda^{2}}\right)^{2},
$$

and we obtain for the cubic and higher-order terms of the Lagrangian,

$$
\hat{\mathcal{L}}^{(n \geq 3)}=\Lambda^{4} \sum_{n=3}^{\infty} \hat{c}_{n}\left(\frac{\partial \hat{\phi}}{\Lambda^{2}}\right)^{n}
$$

with

$$
\hat{c}_{n}=\sum_{m=[n / 2]_{+}}^{n} \bar{K}^{(m)} \bar{X}^{m-n / 2}
$$

where $[n / 2]_{+}$is the smallest integer that is greater than or equal to $n / 2$. In terms of the rescaled field $\varphi$, this gives

$$
\hat{\mathcal{L}}^{(n \geq 3)}=\Lambda^{4} \sum_{n=3}^{\infty} c_{n}\left(\frac{\partial \varphi}{\Lambda^{2}}\right)^{n}
$$

with

$$
c_{n}=\left(\bar{K}^{\prime}+2 \bar{X} \bar{K}^{\prime \prime}\right)^{-n / 2} \sum_{m=[n / 2]_{+}}^{n} \bar{K}^{(m)} \bar{X}^{m-n / 2} .
$$

In the weak-field regime, $\bar{X} \ll 1$, the sum (158) is dominated by the first term $m=[n / 2]_{+}$and even-order terms are of the order of unity while odd-order terms are of the order of $\bar{X}^{1 / 2}$,

$$
\bar{X} \ll 1: c_{2 n} \sim 1 .
$$

On the other hand, in the strong-field regime, $\bar{X} \gg 1$, using $\bar{K}^{(n)} \sim \bar{K} / \bar{X}^{n}$ for the power law $K \propto X^{\nu}$, all terms in the sum (158) contribute and we obtain

$$
\bar{X} \gg 1: c_{n} \sim \bar{K}^{1-n / 2} \ll 1 .
$$

From the propagator (153) and the vertices (157), a typical $L$-loop vacuum Feynman diagram contributing to the corrections to the classical action reads

$I_{L}=\int \prod_{\ell=1}^{L} d^{4} p_{\ell} \prod_{n=1}^{N} \frac{1}{p^{2}+\bar{m}^{2}} \prod_{v=1}^{V} \Lambda^{4} c_{m_{v}} \prod_{s=1}^{m_{v}} \frac{p_{s}}{\Lambda^{2}}$,

where there are $N$ propagators corresponding to $N$ lines in the diagram and $V$ vertices, each with a degree $m_{v}$. Rescaling momenta by $\bar{m}$, using the Euler identity $V-N=$ $1-L$ and $\sum_{v=1}^{V} m_{v}=2 N$, we obtain

$$
I_{L}=\Lambda^{4}\left(\frac{\bar{m}}{\Lambda}\right)^{4 L}\left(\prod_{v=1}^{V} c_{m_{v}}\right) \tilde{I}_{L},
$$

where the integral $\tilde{I}_{L}$ is dimensionless and does not depend on $\bar{m}, \Lambda$, or $\bar{K}$. It is divergent and needs to be regularized, for instance using dimensional regularization. The infinite part appears as poles in $1 /(d-4)$, where $d$ is the dimension of spacetime. Removing these infinities requires introducing counterterms in the bare action. This leaves finite corrections to the classical action that scale as

$$
L \geq 1: \delta \mathcal{L}^{(L)} \sim \Lambda^{4}\left(\frac{\bar{m}}{\Lambda}\right)^{4 L}\left(\prod_{v=1}^{V} c_{m_{v}}\right) .
$$

Notice that this expression depends on the background field via $\bar{m}$, the coefficients $c_{m_{v}}$, and the sound speed $c_{s}$ (which we omit in the expressions). There are two types of corrections. The first ones involve the second term in the expression (149) of the effective mass $\bar{m}$ and depends on higher derivatives $\partial \bar{X}$ and $\partial^{2} \bar{X}$, i.e., second and third derivatives of $\bar{\phi}$. If they were the only types of corrections, we would retrieve the usual nonrenormalization theorem of $K(X)$ theories. The second ones involve the first term only in the expression (149) of $\bar{m}, m^{2} /\left(\bar{K}^{\prime}+2 \bar{X} \bar{K}^{\prime \prime}\right)$, as well as 
factors of $c_{s}$ and $c_{m_{v}}$. These corrections depend on $\bar{X}$ and provide corrections to the classical Lagrangian $K(\bar{X})$. Hence in the models considered here the classical Lagrangian is renormalized, because the bare mass is nonzero. Nevertheless, these quantum corrections can remain negligible, as we now investigate.

First, in the weak-field regime, $\bar{X} \ll 1$, we obtain from (150) and (159)

$$
L \geq 1: \delta \mathcal{L}^{(L)} \sim \Lambda^{4}\left(\frac{m}{\Lambda}\right)^{4 L} .
$$

Therefore, higher loop corrections are under control and become increasingly small at higher orders provided

$$
m \ll \Lambda: \delta \mathcal{L}^{(L)} \ll \delta \mathcal{L}^{(1)} \quad \text { for } L \geq 2 .
$$

We must now compare the leading one-loop term to the classical action, $\mathcal{L}^{(0)}$,

$$
\mathcal{L}^{(0)} \sim \Lambda^{4} \bar{X}-\frac{m^{2}}{2} \bar{\phi}^{2} \sim \bar{\rho}_{\phi} \gtrsim \bar{\rho}_{0},
$$

where $\bar{\rho}_{0}$ is the mean density of the Universe at redshift $z=0$. This gives

$$
m^{4} \ll \bar{\rho}_{0}: \delta \mathcal{L}^{(1)} \ll \mathcal{L}^{(0)}
$$

which reads

$$
m \ll 10^{-3} \mathrm{eV}
$$

Second, in the strong-field regime, $\bar{X} \gg 1$, we obtain from (151) and (160)

$$
L \geq 1: \delta \mathcal{L}^{(L)} \sim \Lambda^{4} \bar{K}\left(\frac{m}{\Lambda}\right)^{4 L}\left(\bar{K} \bar{X}^{-2 / 3}\right)^{-3 L} .
$$

Therefore, higher loop corrections do not blow up provided $\bar{K} \bar{X}^{-2 / 3}$ does not go to 0 at large $\bar{X}$. For the power-law behavior (102) this gives the two conditions

$$
m \ll \Lambda \text { and } \quad \nu \geq \frac{2}{3}: \delta \mathcal{L}^{(L)} \ll \delta \mathcal{L}^{(1)} \text { for } L \geq 2 .
$$

The classical action is now of the order of $\mathcal{L}^{(0)} \sim \Lambda^{4} \bar{K}$. As $\nu \geq 2 / 3$ ensures $\bar{K} \bar{X}^{-2 / 3} \gtrsim 1$ and we have $m \ll \Lambda$, the conditions (170) also give $\delta \mathcal{L}^{(1)} \ll \mathcal{L}^{(0)}$.

Therefore, the quantum corrections remain small for any scalar-field background, in the weak-gravity regime, provided we have the three conditions

$$
m \ll \Lambda, \quad m \ll 10^{-3} \mathrm{eV}, \quad \text { and } \quad \nu \geq \frac{2}{3} \text { at large } X .
$$

The condition $\nu \geq 2 / 3$ is satisfied by the model (109). However, even at the $\mathrm{BH}$ horizon we have $\mu \lesssim 3.5$ from Eq. (116), i.e., $\bar{X} \sim 1$. Therefore, even in this high-density region we do not probe the regime $\bar{X} \gg 1$ and we do not really need to satisfy the asymptotic condition $\nu \geq 2 / 3$ to keep the quantum corrections negligible. From Eq. (7), we note that for $k_{2} \sim 1$ the quantum stability implies $\lambda_{4} \ll 1$.

\section{B. Schwarzschild background metric}

We go beyond the Minkowski spacetime and consider the Schwarzschild metric (9) for the background. This is valid from the large-radius weak-gravity regime (15), which is already covered by the analysis of the previous section, down to the $\mathrm{BH}$ horizon in the strong-gravity regime. The background scalar field $\bar{\phi}(r, t)$ now depends on both radius and time, following the solution (32) described by the nonlinear oscillator $\operatorname{ck}(u, \mu)$. At each radius, the background kinetic argument $\bar{X}$ oscillates with time in the range given in (117).

As in Sec. VII D 2, we work with the background Eddington metric (120), to be able to study the scalar field down to the $\mathrm{BH}$ horizon. We again obtain the Lagrangian of the fluctuations $\hat{\phi}$ from Eq. (142), paying attention to the fact that $\bar{\phi}$ now depends on both time and radius. In particular, using Eqs. (129), (36), and (38), the derivatives of the background solution (32) with respect to the Eddington coordinates read

$$
\frac{\partial \bar{\phi}}{\partial \tilde{t}}=\phi_{0} \omega \frac{\partial \mathrm{ck}}{\partial u}
$$

and

$$
\frac{\partial \bar{\phi}}{\partial \tilde{r}}=\phi_{0}\left[\omega\left(1-\frac{1}{f}\right)-\frac{\mathbf{Q} \beta^{\prime}}{\sqrt{f h}}\right] \frac{\partial \mathrm{ck}}{\partial u}
$$

Notice that $\frac{\partial \bar{\phi}}{\partial \tilde{r}} \neq \frac{\partial \bar{\phi}}{\partial \grave{t}}$ because there are additional $\tilde{r}$-dependent terms to the one explicitly written in Eq. (121), such as the radial dependence of the oscillatory parameter $\mu(r)$. Then, the Lagrangian of the fluctuations $\hat{\phi}$ reads at second order

$$
\begin{aligned}
\hat{\mathcal{L}}^{(2)}= & \frac{1}{2}\left[\mathcal{K}_{00}\left(\frac{\partial \hat{\phi}}{\partial \tilde{t}}\right)^{2}+2 \mathcal{K}_{01} \frac{\partial \hat{\phi}}{\partial \tilde{t}} \frac{\partial \hat{\phi}}{\partial \tilde{r}}+\mathcal{K}_{11}\left(\frac{\partial \hat{\phi}}{\partial \tilde{r}}\right)^{2}\right] \\
& -\frac{\bar{K}^{\prime}}{2 \tilde{r}^{2}}\left(\partial_{\Omega} \hat{\phi}\right)^{2}-\frac{m^{2}}{2} \hat{\phi}^{2}
\end{aligned}
$$

where $\partial_{\Omega} \hat{\phi}$ is the angular derivative, with respect to the longitudinal and azimuthal angles, and the coefficients $\mathcal{K}_{i j}$ are given by 


$$
\begin{gathered}
\mathcal{K}_{00}=(2-f) \bar{K}^{\prime}+\frac{2 \bar{X} \bar{K}^{\prime \prime}}{f^{2} h m^{2}}\left[(f-1)^{2} f\left(\mathbf{Q} \beta^{\prime}\right)^{2}\right. \\
\left.+2 \sqrt{f h}(1-f) \omega \mathbf{Q} \beta^{\prime}+h \omega^{2}\right], \\
\mathcal{K}_{01}=(f-1) \bar{K}^{\prime}+\frac{2 \bar{X} \bar{K}^{\prime \prime}}{f h m^{2}}\left[(1-f) f\left(\mathbf{Q} \beta^{\prime}\right)^{2}+\sqrt{f h} \omega \mathbf{Q} \beta^{\prime}\right], \\
\mathcal{K}_{11}=-f \bar{K}^{\prime}+\frac{2 \bar{X} \bar{K}^{\prime \prime} f\left(\mathbf{Q} \beta^{\prime}\right)^{2}}{h m^{2}} .
\end{gathered}
$$

We recover the scale-independent Minkowski case (144) for $f=h=1, \omega=m$, and $\beta^{\prime}=0$. For $f \neq 1$ or $\beta^{\prime} \neq 0$ we have a mixing of the time and radial derivatives in the kinetic term. Using Eq. (48), we find that the determinant of the kinetic matrix $\mathcal{K}_{i j}$, with $\mathcal{K}_{10}=\mathcal{K}_{01}$, takes the simple form

$$
\operatorname{det}\left(\mathcal{K}_{i j}\right)=-\bar{K}^{\prime}\left(\bar{K}^{\prime}+2 \bar{X} \bar{K}^{\prime \prime}\right)<0 .
$$

Here we assumed that the constraints (145) are already satisfied by the kinetic function $K(X)$. Remarkably, it coincides with the determinant obtained in the Minkowski case (144) as it does not depend on the metric potentials $f$ and $h$, or on $\beta^{\prime}$, but only on the properties of the kinetic function $K(X)$. Its negative sign implies that the quadratic form governing the kinetic terms in the $(\tilde{t}, \tilde{r})$ plane has two opposite-sign eigenvalues $\lambda_{+}>0>\lambda_{-}$. This always preserves the signature $(+,-)$ and guarantees the absence of ghost and gradient instability. Moreover, as $\operatorname{det}\left(\mathcal{K}_{i j}\right)$ does not vanish the two branches $\lambda_{+}(x)$ and $\lambda_{-}(x)$ are well separated and do not make contact. Therefore, the positive eigenvalue is connected to the eigenvector $\frac{\partial \bar{\phi}}{\partial \grave{t}}$ at large radii, while the negative eigenvalue is connected to the eigenvector $\frac{\partial \bar{\phi}}{\partial \tilde{r}}$. Close to the horizon, the eigenvectors are a linear combination of $\frac{\partial \bar{\phi}}{\partial \bar{t}}$ and $\frac{\partial \bar{\phi}}{\partial \tilde{r}}$. However, one could define new time and radial coordinates $\hat{t}$ and $\hat{r}$, from linear combinations of $\tilde{t}$ and $\tilde{r}$, so that $\hat{t}$ and $\hat{r}$ converge to $\tilde{t}$ and $\tilde{r}$ at large radii and the kinetic term takes the diagonal form $\frac{1}{2}\left[\lambda_{+}\left(\frac{\partial \bar{\phi}}{\partial \hat{t}}\right)^{2}+\lambda_{-}\left(\frac{\partial \bar{\phi}}{\partial \hat{r}}\right)^{2}\right]$. We can check this behavior in Fig. 9, where we consider the two boundaries 0 and $X_{\max }$ of Eq. (117) of the range spanned by the oscillating background $\bar{X}(r, t)$. Therefore, the second-order Lagrangian $\hat{\mathcal{L}}^{(2)}$ can be the basis of a well-defined quantum perturbation theory.

Because of the nondiagonal kinetic matrix $\mathcal{K}_{i j}$, the propagator is different from the Minkowski rescaled propagator (153). However, by going for instance to the diagonal coordinates $\{\hat{t}, \hat{r}\}$ and using the fact that $\lambda_{ \pm}$ remain of order unity, the scalings that we obtained in the previous section VIII A in the regime $\bar{X} \sim 1$ and $\bar{K}^{\prime} \sim 1$ remain valid. In particular, as in Eq. (153) each propagator

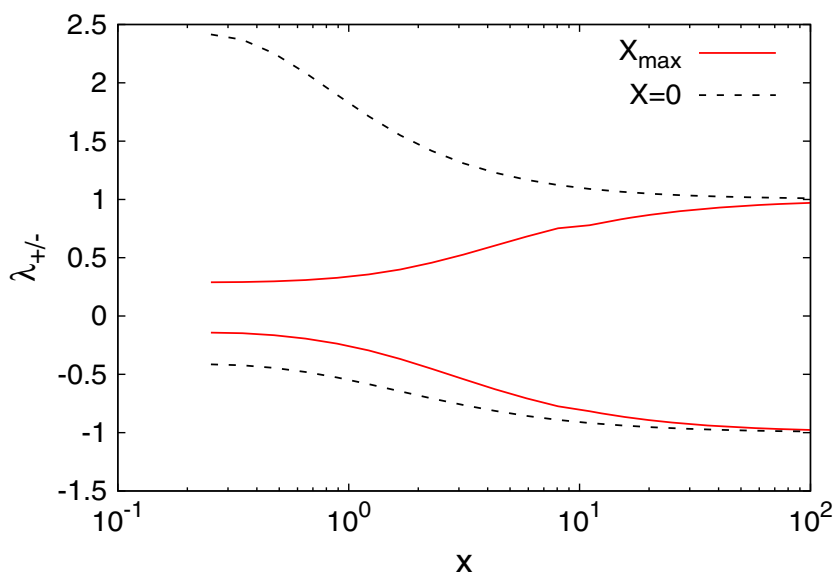

FIG. 9. Radial profile of the eigenvalues $\lambda_{+}>0>\lambda_{-}$of the kinetic matrix $\mathcal{K}_{i j}$ that appears in Eq. (174). We show the results obtained for $\bar{X}=X_{\max }$ (solid lines) and for $\bar{X}=0$ (dashed lines).

brings a factor $1 / \mathrm{m}^{2}$ and as in Eq. (155) vertices take the form $\Lambda^{4}\left(\partial \hat{\phi} / \Lambda^{2}\right)^{n}$, with coefficients $c_{n}$ of the order of unity. Therefore, the power counting of loop diagrams is not altered and we recover Eq. (164), while the classical Lagrangian is now of order $\mathcal{L}^{(0)} \sim \Lambda^{4} \bar{K} \sim \Lambda^{4}$ as $\bar{K} \sim 1$. Then, quantum loop corrections are small provided $m \ll \Lambda$,

$$
m \ll \Lambda: \delta \mathcal{L}^{(L)} \ll \mathcal{L}^{(0)}
$$

This condition was already required in (165) for the Minkowski background; therefore, the classical analysis developed in previous sections remains valid down to the horizon.

Finally notice that the strong-coupling scale $\Lambda$ is not the cutoff of the quantum theory. Indeed, nothing prevents one from using classical backgrounds where $\rho_{\phi} \sim \Lambda^{4}$ as long as the quantum corrections are under control, i.e., as long as the conditions (171) are satisfied.

\section{CONCLUSION}

We have shown in a previous article that a scalar field with a nonstandard kinetic term can play the role of dark matter in the late Universe and build static solitonic profiles in galaxies, with a flat core. In this weak-gravity and weakfield regime, the first quartic correction $-(\partial \phi)^{4}$ to the kinetic term is the dominant subleading correction. It provides an effective pressure that balances the self-gravity of the scalar cloud and gives rise to a static equilibrium.

In this paper, we have investigated the impact of a supermassive $\mathrm{BH}$ at the center of galaxies on this scenario. To this order, following the spirit of our previous work for the case of a quartic subleading potential $\phi^{4}$, which also gives rise to an effective pressure in the weak-gravity and weak-field regime, we have obtained the explicit solution of the scalar-field equation of motion in the large scalar 
mass limit. For a $\phi^{4}$ correction to the potential, the nonlinearity transformed the usual harmonic wave solution of the standard Klein-Gordon equation into a nonlinear wave described by the Jacobi elliptic function $\operatorname{cn}(u, k)$. In a similar fashion, we show how arbitrary kinetic functions $K(X)$ lead to associated nonlinear oscillatory functions $\operatorname{ck}(u, \mu)$, which extend the harmonic $\operatorname{cosine} \cos (u)$ and the Jacobi elliptic function $\mathrm{cn}(u, k)$. They correspond to second-order ordinary differential equations with nonlinear derivative terms, which reduce to the harmonic oscillator at linear order. In the large-mass limit, the scalar field shows fast oscillations with time, with an angular frequency $\omega$ of order $m$, with an amplitude and a phase that show a slow dependence on the distance from the central $\mathrm{BH}$.

Contrary to the case of a quartic potential $\phi^{4}$, we find that the quartic derivative self-interaction $-(\partial \phi)^{4}$ is not able to support the scalar cloud down to the $\mathrm{BH}$ horizon, in the relativistic regime. A continuous solution can only describe the late stage of the infall, when most of the scalar-field energy density has already fallen into the $\mathrm{BH}$. Therefore, such models cannot provide realistic dark-matter scenarios. This shows the importance of going beyond the weakgravity large-radius analysis and of studying the selfconsistency of the system down to the horizon, in the relativistic regime and strong-gravity regime.

We discussed the generic conditions to obtain a wellbehaved global solution. We obtain the usual conditions $K^{\prime}>0$ and $K^{\prime}+2 X K^{\prime \prime}>0$, which are typically associated with the stability of perturbations in $k$-essence models, i.e., the absence of ghost and the positivity of the squared speed of sound. We also note that in order to have a global solution, which satisfies the boundary condition at the horizon and converges to the static soliton at large radii, $K(X)$ must typically grow as a power law $X^{\nu}$ with $1 / 2<$ $\nu<1$ at large $X$.

We have presented a detailed analysis of a simple wellbehaved example, $K(X)=(1+3 X / 2)^{2 / 3}-1$. There, in a fashion similar to both the hydrodynamical case and the quartic potential case, a unique global solution exists. It is associated with a critical value of the flux that allows the solution to match the boundary conditions at both small and large radii. This solution is well defined down to the horizon, once we use appropriate coordinates such as the Eddington time. The amplitude of the scalar field $\phi$ and of the kinetic argument $X$ remain finite at the Schwarzschild radius. It leads to a slow infall of the scalar cloud into the $\mathrm{BH}$, as the radial velocity grows from a negligible value at large radii, in the quasistatic solitonic regime, to relativistic values that follow the free infall at the horizon. The infall timescale is much greater than the age of the Universe; hence these models can provide realistic scenarios for the dark-matter galactic halos.

Finally, we investigated the importance of quantum corrections to the classical action. We obtained the conditions for these quantum corrections to remain negligible in the configurations that we study in this paper, both in the weak-gravity regime, well described by the Minkowski background metric, and the strong-gravity regime, described by the Schwarzschild metric. We recover the usual constraints $K^{\prime}>0$ and $K^{\prime}+2 X K^{\prime \prime}>0$ for a well-behaved setup, and we find that when $m \ll \Lambda$ and $m \ll 10^{-3} \mathrm{eV}$ the quantum corrections remain small. This holds both for small and large scalar-field values, which can probe the strongly coupled regime [there, in addition, $K(X)$ must typically grow as $X^{\nu}$ with $\left.2 / 3 \leq \nu<1\right]$.

Thus, we find that scalar fields with nonstandard kinetic terms can provide realistic models of dark matter, building solitonic cores at galactic centers that are stable over the age of the Universe. Moreover, the quantum corrections remain well under control. The conditions on the kinetic function $K(X)$ are mostly the standard constraints, $K^{\prime}>0$ and $K^{\prime}+2 X K^{\prime \prime}>0$, with the addition of a more subtle condition associated with the existence of a global solution, which roughly corresponds to $K(X) \sim X^{\nu}$ with $2 / 3 \leq \nu<1$ over $1 \lesssim X \lesssim 50$. The scalar mass must obey $m \ll 10^{-3} \mathrm{eV}$ while the strong-coupling scale must verify $\Lambda \gg m$.

\section{ACKNOWLEDGMENTS}

This work is supported in part by the EU Horizon 2020 research and innovation program under the MarieSklodowska Grant No. 690575. This article is based upon work related to the European Cooperation in Science and Technology (COST) Action Grant No. CA15117 (CANTATA) supported by COST. The work by J. A. R. C. is partially supported by the MINECO (Spain) Grant No. FIS2016-78859-P(AEI/FEDER, UE).
[1] J. P. Ostriker and P. J. Steinhardt, Science 300, 1909 (2003).

[2] D. H. Weinberg, J. S. Bullock, F. Governato, R. Kuzio de Naray, and A. H. G. Peter, Proc. Nat. Acad. Sci. 112, 12249 (2015)
[3] A. Pontzen and F. Governato, Nature (London) 506, 171 (2014).

[4] W. J. G. de Blok, Adv. Astron. 2010, 1 (2010).

[5] B. Moore, S. Ghigna, F. Governato, G. Lake, T. R. Quinn, J. Stadel, and P. Tozzi, Astrophys. J. 524, L19 (1999). 
[6] M. Boylan-Kolchin, J. S. Bullock, and M. Kaplinghat, Mon. Not. R. Astron. Soc. 415, L40 (2011).

[7] R. D. Peccei and H. R. Quinn, Phys. Rev. Lett. 38, 1440 (1977).

[8] F. Wilczek, Phys. Rev. Lett. 40, 279 (1978).

[9] S. Weinberg, Phys. Rev. Lett. 40, 223 (1978).

[10] D. J. E. Marsh, Phys. Rep. 643, 1 (2016).

[11] W. Hu, R. Barkana, and A. Gruzinov, Phys. Rev. Lett. 85, 1158 (2000).

[12] L. Hui, J. P. Ostriker, S. Tremaine, and E. Witten, Phys. Rev. D 95, 043541 (2017).

[13] M. C. Johnson and M. Kamionkowski, Phys. Rev. D 78, 063010 (2008).

[14] J.-c. Hwang and H. Noh, Phys. Lett. B 680, 1 (2009).

[15] C.-G. Park, J.-c. Hwang, and H. Noh, Phys. Rev. D 86, 083535 (2012).

[16] R. Hlozek, D. Grin, D. J. E. Marsh, and P. G. Ferreira, Phys. Rev. D 91, 103512 (2015).

[17] J. A. R. Cembranos, A. L. Maroto, and S. J. Núñez Jareño, J. High Energy Phys. 03 (2016) 013.

[18] J. A. R. Cembranos, A. L. Maroto, and S. J. Núñez Jareño, J. High Energy Phys. 02 (2017) 064.

[19] H.-Y. Schive, T. Chiueh, and T. Broadhurst, Nat. Phys. 10, 496 (2014).

[20] T. Broadhurst, H. N. Luu, and S.H.H. Tye, arXiv: 1811.03771.

[21] J. A. R. Cembranos, J. L. Feng, A. Rajaraman, and F. Takayama, Phys. Rev. Lett. 95, 181301 (2005).

[22] P.-H. Chavanis, Phys. Rev. D 84, 043531 (2011).
[23] P. H. Chavanis and L. Delfini, Phys. Rev. D 84, 043532 (2011).

[24] J. A. R. Cembranos, A. L. Maroto, S. J. Núñez Jareño, and H. Villarrubia-Rojo, J. High Energy Phys. 08 (2018) 073.

[25] P.-H. Chavanis, Phys. Rev. D 100, 083022 (2019).

[26] E. Armengaud, N. Palanque-Delabrouille, C. Yche, D. J. E. Marsh, and J. Baur, Mon. Not. R. Astron. Soc. 471, 4606 (2017).

[27] P. Brax, J. A. R. Cembranos, and P. Valageas, Phys. Rev. D 100, 023526 (2019).

[28] M. Khlopov, B. A. Malomed, and I. B. Zeldovich, Mon. Not. R. Astron. Soc. 215, 575 (1985).

[29] J. Goodman, New Astron. 5, 103 (2000).

[30] B. Li, T. Rindler-Daller, and P. R. Shapiro, Phys. Rev. D 89, 083536 (2014).

[31] A. Suárez and P.-H. Chavanis, Phys. Rev. D 95, 063515 (2017).

[32] A. Suárez and P.-H. Chavanis, Phys. Rev. D 92, 023510 (2015).

[33] P. Brax, P. Valageas, and J. A. R. Cembranos, Phys. Rev. D 101, 023521 (2020).

[34] C. Armendariz-Picon, V. F. Mukhanov, and P. J. Steinhardt, Phys. Rev. D 63, 103510 (2001).

[35] M. Blau, Lecture notes on general relativity, http://www .blau.itp.unibe.ch/newlecturesGR.pdf (2019).

[36] E. Madelung, Z. Phys. 40, 322 (1927).

[37] F. C. Michel, Astrophys. Space Sci. 15, 153 (1972).

[38] S. L. Shapiro and S. A. Teukolsky, black holes, White Dwarfs, and Neutron Stars: The Physics of Compact Objects (Wiley, New York, USA, 1983), ISBN:9780471873167. 